\title{
On Frobenius-destabilized rank-2 vector bundles over curves
}

Herbert Lange and Christian Pauly

\begin{abstract}
Let $X$ be a smooth projective curve of genus $g \geq 2$ over an algebraically closed field $k$ of characteristic $p>0$. Let $\mathcal{M}_{X}$ be the moduli space of semistable rank-2 vector bundles over $X$ with trivial determinant. The relative Frobenius map $F: X \rightarrow X_{1}$ induces by pull-back a rational map $V: \mathcal{M}_{X_{1}} \rightarrow-\mathcal{M}_{X}$. In this paper we show the following results.

(1) For any line bundle $L$ over $X$, the rank- $p$ vector bundle $F_{*} L$ is stable.

(2) The rational map $V$ has base points, i.e., there exist stable bundles $E$ over $X_{1}$ such that $F^{*} E$ is not semistable.

(3) Let $\mathcal{B} \subset \mathcal{M}_{X_{1}}$ denote the scheme-theoretical base locus of $V$. If $g=2, p>2$ and $X$ ordinary, then $\mathcal{B}$ is a 0 -dimensional local complete intersection of length $\frac{2}{3} p\left(p^{2}-1\right)$ and the degree of $V$ equals $\frac{1}{3} p\left(p^{2}+2\right)$.
\end{abstract}

Mathematics Subject Classification (2000). Primary 14H60, 14D20, Secondary 14H40.

Keywords. Vector bundle, Frobenius map, semi-stability, moduli space.

\section{Introduction}

Let $X$ be a smooth projective curve of genus $g \geq 2$ over an algebraically closed field $k$ of characteristic $p>0$. Denote by $F: X \rightarrow X_{1}$ the relative $k$-linear Frobenius map. Here $X_{1}=X \times_{k, \sigma} k$, where $\sigma: \operatorname{Spec}(k) \rightarrow \operatorname{Spec}(k)$ is the Frobenius of $k$ (see e.g. [R], Section 4.1). We denote by $\mathcal{M}_{X}$, respectively $\mathcal{M}_{X_{1}}$, the moduli space of semistable rank-2 vector bundles on $X$, respectively $X_{1}$, with trivial determinant. The Frobenius $F$ induces by pull-back a rational map (the Verschiebung)

$$
V: \mathcal{M}_{X_{1}} \rightarrow \mathcal{M}_{X}, \quad[E] \mapsto\left[F^{*} E\right] .
$$

Here $[E]$ denotes the $S$-equivalence class of the semistable bundle $E$. It is shown [MS] that $V$ is generically étale, hence separable and dominant, if $X$ or equivalently $X_{1}$ is an ordinary curve. Our first result is

Theorem 1. Over any smooth projective curve $X_{1}$ of genus $g \geq 2$ there exist stable rank-2 vector bundles $E$ with trivial determinant, such that $F^{*} E$ is not semistable. In other words, $V$ has base points. 
Note that this is a statement for an arbitrary curve of genus $g \geq 2$ over $k$, since associating $X_{1}$ to $X$ induces an automorphism of the moduli space of curves of genus $g$ over $k$. The existence of Frobenius-destabilized bundles was already proved in [LP2], Theorem A.4, by specializing the so-called Gunning bundle on a MumfordTate curve. The proof given in this paper is much simpler than the previous one. Given a line bundle $L$ over $X$, the generalized Nagata-Segre theorem asserts the existence of rank-2 subbundles $E$ of the rank- $p$ bundle $F_{*} L$ of a certain (maximal) degree. Quite surprisingly, these subbundles $E$ of maximal degree turn out to be stable and Frobenius-destabilized.

In the case $g=2$ the moduli space $\mathcal{M}_{X}$ is canonically isomorphic to the projective space $\mathbb{P}_{k}^{3}$ and the set of strictly semistable bundles can be identified with the Kummer surface $\operatorname{Kum}_{X} \subset \mathbb{P}_{k}^{3}$ associated to $X$. According to [LP2], Proposition A.2, the rational map

$$
V: \mathbb{P}_{k}^{3--\rightarrow} \mathbb{P}_{k}^{3}
$$

is given by polynomials of degree $p$, which are explicitly known in the cases $p=2$ [LP1] and $p=3$ [LP2]. Let $\mathscr{B}$ be the scheme-theoretical base locus of $V$, i.e., the subscheme of $\mathbb{P}_{k}^{3}$ determined by the ideal generated by the 4 polynomials of degree $p$ defining $V$. Clearly its underlying set equals (see [O1], Theorem A.6)

$$
\operatorname{supp} \mathscr{B}=\left\{E \in \mathcal{M}_{X_{1}} \cong \mathbb{P}_{k}^{3} \mid F^{*} E \text { is not semistable }\right\}
$$

and supp $\mathscr{B} \subset \mathbb{P}_{k}^{3} \backslash \operatorname{Kum}_{X_{1}}$. Since $V$ has no base points on the ample divisor $\operatorname{Kum}_{X_{1}}$, we deduce that $\operatorname{dim} \mathscr{B}=0$. Then we show

Theorem 2. Assume $p>2$. Let $X_{1}$ be an ordinary curve of genus $g=2$. Then the 0 -dimensional scheme $B$ is a local complete intersection of length

$$
\frac{2}{3} p\left(p^{2}-1\right)
$$

Since $\mathscr{B}$ is a local complete intersection, the degree of $V$ equals deg $V=p^{3}-l(\mathscr{B})$ where $l(\mathscr{B})$ denotes the length of $\mathscr{B}$ (see e.g. [O1], Proposition 2.2). Hence we obtain the

Corollary. Under the assumption of Theorem 2

$$
\operatorname{deg} V=\frac{1}{3} p\left(p^{2}+2\right) .
$$

The underlying idea of the proof of Theorem 2 is rather simple: we observe that

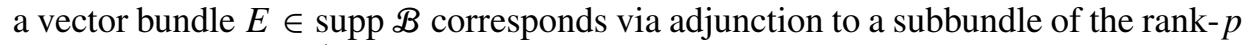
vector bundle $F_{*}\left(\theta^{-1}\right)$ for some theta characteristic $\theta$ on $X$ (Proposition 3.1). This is the motivation to introduce Grothendieck's Quot-scheme $\mathcal{Q}$ parametrizing rank-2 
subbundles of degree 0 of the vector bundle $F_{*}\left(\theta^{-1}\right)$. We prove that the two 0 dimensional schemes $\mathscr{B}$ and $\mathcal{Q}$ decompose as disjoint unions $\bigsqcup \mathcal{B}_{\theta}$ and $\bigsqcup \mathcal{Q}_{\eta}$ where $\theta$ and $\eta$ vary over theta characteristics on $X$ and $p$-torsion points of $J X_{1}$ respectively and that $\mathscr{B}_{\theta}$ and $\mathcal{Q}_{0}$ are isomorphic, if $X$ is ordinary (Proposition 4.6). In particular since $\mathcal{Q}$ is a local complete intersection, $\mathscr{B}$ also is.

In order to compute the length of $\mathscr{B}$ we show that $\mathcal{Q}$ is isomorphic to a determinantal scheme $\mathscr{D}$ defined intrinsically by the 4 -th Fitting ideal of some sheaf. The non-existence of a universal family over the moduli space of rank- 2 vector bundles of degree 0 forces us to work over a different parameter space constructed via the Hecke correspondence and carry out the Chern class computations on this parameter space.

The underlying set of points of $\mathscr{B}$ has already been studied in the literature. In fact, using the notion of $p$-curvature, S. Mochizuki $[\mathrm{Mo}]$ describes points of $\mathscr{B}$ as "dormant atoms" and obtains, by degenerating the genus- 2 curve $X$ to a singular curve, the above mentioned formula for their number ([Mo], Corollary 3.7, p. 267).

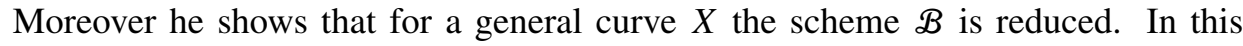
context we also mention the recent work of B. Osserman [O1], [O2], which explains the relationship of supp $\mathbb{B}$ with Mochizuki's theory.

Acknowledgments. We would like to thank Yves Laszlo and Brian Osserman for helpful discussions and for pointing out several mistakes in a previous version of this paper. We also thank Adrian Langer for some advice with references. We are also grateful to the referee for interesting comments.

\section{Stability of the direct image $F_{*} L$}

Let $X$ be a smooth projective curve of genus $g \geq 2$ over an algebraically closed field of characteristic $p>0$ and let $F: X \rightarrow X_{1}$ denote the relative Frobenius map. Let $L$ be a line bundle over $X$ with

$$
\operatorname{deg} L=g-1+d,
$$

for some integer $d$. Applying the Grothendieck-Riemann-Roch theorem to the morphism $F$, we obtain

Lemma 1.1. The slope of the rank-p vector bundle $F_{*} L$ equals

$$
\mu\left(F_{*} L\right)=g-1+\frac{d}{p} .
$$

The following result will be used in Section 3 . 
Proposition 1.2. If $g \geq 2$, then the vector bundle $F_{*} L$ is stable for any line bundle Lon $X$.

Proof. Suppose that the contrary holds, i.e., $F_{*} L$ is not stable. Consider its HarderNarasimhan filtration

$$
0=E_{0} \subset E_{1} \subset E_{2} \subset \cdots \subset E_{l}=F_{*} L,
$$

such that the quotients $E_{i} / E_{i-1}$ are semistable with $\mu\left(E_{i} / E_{i-1}\right)>\mu\left(E_{i+1} / E_{i}\right)$ for all $i \in\{1, \ldots, l-1\}$. If $F_{*} L$ is not semistable, we denote $E:=E_{1}$. If $F_{*} L$ is semistable, we denote by $E$ any proper semistable subbundle of the same slope. Then clearly

$$
\mu(E) \geq \mu\left(F_{*} L\right) .
$$

In case $r=\operatorname{rk} E>\frac{p-1}{2}$, we observe that the quotient bundle

$$
Q= \begin{cases}F_{*} L / E_{l-1} & \text { if } F_{*} L \text { is not semistable } \\ F_{*} L / E & \text { if } F_{*} L \text { is semistable }\end{cases}
$$

is also semistable and that its dual $Q^{*}$ is a subbundle of $\left(F_{*} L\right)^{*}$. Moreover, by relative duality $\left(F_{*} L\right)^{*}=F_{*}\left(L^{-1} \otimes \omega_{X}^{\otimes 1-p}\right)$ and by assumption rk $Q^{*} \leq p-r \leq \frac{p-1}{2}$. Hence, replacing if necessary $E$ and $L$ by $Q^{*}$ and $L^{-1} \otimes \omega_{X}^{\otimes 1-p}$, we may assume that $E$ is semistable and $r \leq \frac{p-1}{2}$.

Now, by [SB], Corollary 2, we have the inequality

$$
\mu_{\max }\left(F^{*} E\right)-\mu_{\min }\left(F^{*} E\right) \leq(r-1)(2 g-2),
$$

where $\mu_{\max }\left(F^{*} E\right)$ (resp. $\left.\mu_{\min }\left(F^{*} E\right)\right)$ denotes the slope of the first (resp. last) graded piece of the Harder-Narasimhan filtration of $F^{*} E$. The inclusion $E \subset F_{*} L$ gives, by adjunction, a nonzero map $F^{*} E \rightarrow L$. Hence

$\operatorname{deg} L \geq \mu_{\min }\left(F^{*} E\right) \geq \mu_{\max }\left(F^{*} E\right)-(r-1)(2 g-2) \geq p \mu(E)-(r-1)(2 g-2)$.

Combining this inequality with (1) and using Lemma 1.1, we obtain

$$
g-1+\frac{d}{p}=\mu\left(F_{*} L\right) \leq \mu(E) \leq \frac{g-1+d}{p}+\frac{(r-1)(2 g-2)}{p},
$$

which simplifies to

$$
(g-1) \leq(g-1)\left(\frac{2 r-1}{p}\right) .
$$

This is a contradiction, since we have assumed $r \leq \frac{p-1}{2}$ and therefore $\frac{2 r-1}{p}<1$. 
Remark 1.3. We observe that the vector bundles $F_{*} L$ are destabilized by Frobenius, because of the nonzero canonical map $F^{*} F_{*} L \rightarrow L$ and clearly $\mu\left(F^{*} F_{*} L\right)>\operatorname{deg} L$. For further properties of the bundles $F_{*} L$, see [JRXY], Section 5.

Remark 1.4. In the context of Proposition 1.2 we mention the following open question: given a finite separable morphism between smooth curves $f: Y \rightarrow X$ and a line bundle $L \in \operatorname{Pic}(Y)$, is the direct image $f_{*} L$ stable? For a discussion, see [B2].

\section{Existence of Frobenius-destabilized bundles}

Let the notation be as in the previous section. We recall the generalized Nagata-Segre theorem, proved by Hirschowitz, which says

Theorem 2.1. For any vector bundle $G$ of rank $r$ and degree $\delta$ over any smooth curve $X$ and for any integer $n, 1 \leq n \leq r-1$, there exists a rank-n subbundle $E \subset G$, satisfying

$$
\mu(E) \geq \mu(G)-\left(\frac{r-n}{r}\right)(g-1)-\frac{\varepsilon}{r n},
$$

where $\varepsilon$ is the unique integer with $0 \leq \varepsilon \leq r-1$ and $\varepsilon+n(r-n)(g-1) \equiv n \delta \bmod r$.

Remark 2.2. The previous theorem can be deduced (see [L], Remark 3.14) from the main theorem of [Hir] (for its proof, see http://math.unice.fr/ ah/math/Brill/).

Proof of Theorem 1. We apply Theorem 2.1 to the rank- $p$ vector bundle $F_{*} L$ on $X_{1}$ and $n=2$, where $L$ is a line bundle of degree $g-1+d$ on $X$, with $d \equiv-2 g+2 \bmod p$ : There exists a rank-2 vector bundle $E \subset F_{*} L$ such that

$$
\mu(E) \geq \mu\left(F_{*} L\right)-\frac{p-2}{p}(g-1) .
$$

Note that our assumption on $d$ was made to have $\varepsilon=0$.

Now we will check that any $E$ satisfying inequality (4) is stable with $F^{*} E$ not semistable.

(i) $E$ is stable: Let $N$ be a line subbundle of $E$. The inclusion $N \subset F_{*} L$ gives, by adjunction, a nonzero map $F^{*} N \rightarrow L$, which implies (see also [JRXY], Proposition 3.2 (i))

$$
\operatorname{deg} N \leq \mu\left(F_{*} L\right)-\frac{p-1}{p}(g-1) .
$$

Comparing with (4) we see that $\operatorname{deg} N<\mu(E)$. 
(ii) $F^{*} E$ is not semistable. In fact, we claim that $L$ destabilizes $F^{*} E$. For the proof note that Lemma 1.1 implies

$$
\mu\left(F_{*} L\right)-\frac{p-2}{p}(g-1)=\frac{2 g-2+d}{p}>\frac{g-1+d}{p}=\frac{\operatorname{deg} L}{p}
$$

since $g \geq 2$. Together with (4) this gives $\mu(E)>\frac{\operatorname{deg} L}{p}$ and hence

$$
\mu\left(F^{*} E\right)>\operatorname{deg} L .
$$

This implies the assertion, since by adjunction we obtain a nonzero map $F^{*} E \rightarrow L$.

Replacing $E$ by a subsheaf of suitable degree, we may assume that inequality (4) is an equality. In that case, because of our assumption on $d, \mu(E)$ is an integer, hence $\operatorname{deg} E$ is even. In order to get trivial determinant, we may tensorize $E$ with a suitable line bundle.

This shows the existence of a stable rank-2 vector bundle $E$ with $F^{*} E$ not semistable, which is equivalent to the existence of base points of $V$ (see e.g. [O1], Theorem A.6).

\section{Frobenius-destabilized bundles in genus 2.}

From now on we assume that $X$ is an ordinary curve of genus $g=2$ and the characteristic of $k$ is $p>2$. Recall that $\mathcal{M}_{X}$ denotes the moduli space of semistable rank-2 vector bundles with trivial determinant over $X$ and $\mathscr{B}$ the scheme-theoretical base locus of the rational map

$$
V: \mathcal{M}_{X_{1}} \cong \mathbb{P}_{k}^{3} \rightarrow \mathbb{P}_{k}^{3} \cong \mathcal{M}_{X},
$$

which is given by polynomials of degree $p$.

First of all we will show that the 0-dimensional scheme $\mathscr{B}$ is the disjoint union of subschemes $\mathscr{B}_{\theta}$ indexed by theta characteristics of $X$.

Proposition 3.1. (a) Let $E$ be a vector bundle on $X_{1}$ such that $E \in \operatorname{supp} \mathscr{B}$. Then we have

(i) There exists a unique theta characteristic $\theta$ on $X$ such that $\operatorname{Hom}\left(E, F_{*}\left(\theta^{-1}\right)\right) \neq$ 0.

(ii) Any rank-2 vector bundle $E$ of degree 0 satisfying $\operatorname{Hom}\left(E, F_{*}\left(\theta^{-1}\right)\right) \neq 0$ is a subbundle of $F_{*}\left(\theta^{-1}\right)$, i.e. the quotient $F_{*}\left(\theta^{-1}\right) / E$ is torsion free.

(b) Let $\theta$ be a theta characteristic on $X$. Any rank-2 subbundle $E \subset F_{*}\left(\theta^{-1}\right)$ of degree 0 has the following properties

(i) $E$ is stable and $F^{*} E$ is not semistable, 
(ii) $F^{*}(\operatorname{det} E)=\mathcal{O}_{X}$,

(iii) $\operatorname{dim} \operatorname{Hom}\left(E, F_{*}\left(\theta^{-1}\right)\right)=1$ and $\operatorname{dim} H^{1}\left(E^{*} \otimes F_{*}\left(\theta^{-1}\right)\right)=5$,

(iv) $E$ is a rank-2 subbundle of maximal degree.

Proof. (a) By [LS], Corollary 2.6, we know that, for every $E \in \operatorname{supp} \mathscr{B}$ the bundle $F^{*} E$ is the nonsplit extension of $\theta^{-1}$ by $\theta$, for some theta characteristic $\theta$ on $X$ (note that $\operatorname{Ext}^{1}\left(\theta^{-1}, \theta\right) \cong k$ ). By adjunction we get a nonzero homomorphism $\psi: E \rightarrow F_{*}\left(\theta^{-1}\right)$, which shows (i). Uniqueness of $\theta$ will be proved below.

As for (ii), we have to show that $\psi$ is of maximal rank. Suppose it is not, then there is a line bundle $N$ on the curve $X_{1}$ such that $\psi$ factorizes as $E \rightarrow N \rightarrow F_{*}\left(\theta^{-1}\right)$. By stability of $E$ we have $\operatorname{deg} N>0$. On the other hand, by adjunction, we get a nonzero homomorphism $F^{*} N \rightarrow \theta^{-1}$ implying $p \cdot \operatorname{deg} N \leq-1$, a contradiction. Hence $\psi: E \rightarrow F_{*}\left(\theta^{-1}\right)$ is injective. Moreover $E$ is even a subbundle of $F_{*}\left(\theta^{-1}\right)$, since otherwise there exists a subbundle $E^{\prime} \subset F_{*}\left(\theta^{-1}\right)$ with $\operatorname{deg} E^{\prime}>0$ and which fits into the exact sequence

$$
0 \longrightarrow E \longrightarrow E^{\prime} \stackrel{\pi}{\longrightarrow} T \longrightarrow 0,
$$

where $T$ is a torsion sheaf supported on an effective divisor. Varying $\pi$, we obtain a family of bundles ker $\pi \subset E^{\prime}$ of dimension $>0$ and det ker $\pi=\mathcal{O}_{X_{1}}$. This would imply (see proof of Theorem 1 ) $\operatorname{dim} \mathscr{B}>0$, a contradiction.

Finally, since $\theta$ is the maximal destabilizing line subbundle of $F^{*} E$, it is unique.

(b) We observe that inequality (4) holds for the pair $E \subset F_{*}\left(\theta^{-1}\right)$. Hence, by the proof of Theorem 1, $E$ is stable and $F^{*} E$ is not semistable.

Let $\varphi: F^{*} E \rightarrow \theta^{-1}$ denote the homomorphism adjoint to the inclusion $E \subset$ $F_{*}\left(\theta^{-1}\right)$. The homomorphism $\varphi$ is surjective, since otherwise $F^{*} E$ would contain a line subbundle of degree $>1$, contradicting [LS], Satz 2.4. Hence we get an exact sequence

$$
0 \rightarrow \operatorname{ker} \varphi \rightarrow F^{*} E \rightarrow \theta^{-1} \rightarrow 0 .
$$

On the other hand, let $N$ denote a line bundle on $X_{1}$ such that $E \otimes N$ has trivial determinant, i.e. $N^{-2}=\operatorname{det} E$. Applying Corollary 2.6 in [LS] to the bundle $F^{*}(E \otimes N)$ we get an exact sequence

$$
0 \rightarrow \tilde{\theta} \otimes F^{*} N^{-1} \rightarrow F^{*} E \rightarrow \tilde{\theta}^{-1} \otimes F^{*} N^{-1} \rightarrow 0,
$$

for some theta characteristic $\tilde{\theta}$. By uniqueness of the destabilizing subbundle of maximal degree of $F^{*} E$, this exact sequence must coincide with (6) up to a nonzero constant. This implies that $F^{*} N \otimes \tilde{\theta}=\theta$, hence $\left(F^{*} N\right)^{2}=\mathcal{O}_{X}$. So we obtain that $\mathcal{O}_{X}=\operatorname{det}\left(F^{*} E\right)=F^{*}(\operatorname{det} E)$ proving (ii).

By adjunction the equality $\operatorname{dim} \operatorname{Hom}\left(E, F_{*}\left(\theta^{-1}\right)\right)=\operatorname{dim} \operatorname{Hom}\left(F^{*} E, \theta^{-1}\right)=1$ holds. Moreover by Riemann-Roch we obtain $\operatorname{dim} H^{1}\left(E^{*} \otimes F_{*}\left(\theta^{-1}\right)\right)=5$. This proves (iii). 
Finally, suppose that there exists a rank-2 subbundle $E^{\prime} \subset F_{*}\left(\theta^{-1}\right)$ with $\operatorname{deg} E^{\prime} \geq 1$. Then we can consider the kernel $E=\operatorname{ker} \pi$ of a surjective morphism $\pi: E^{\prime} \rightarrow T$ onto a torsion sheaf with length equal to deg $E^{\prime}$. By varying $\pi$ and after tensoring ker $\pi$ with a suitable line bundle of degree 0 , we construct a family of dimension $>0$ of stable rank- 2 vector bundles with trivial determinant which are Frobenius-destabilized, contradicting $\operatorname{dim} \mathscr{B}=0$. This proves (iv).

It follows from Proposition 3.1 (a) that the scheme $\mathcal{B}$ decomposes as a disjoint union

$$
\mathscr{B}=\coprod_{\theta} \mathscr{B}_{\theta},
$$

where $\theta$ varies over the set of all theta characteristics of $X$ and

$$
\operatorname{supp} \mathscr{B}_{\theta}=\left\{E \in \operatorname{supp} \mathscr{B} \mid E \subset F_{*}\left(\theta^{-1}\right)\right\} .
$$

Tensor product with a 2-torsion point $\alpha \in J X_{1}[2] \cong J X[2]$ induces an isomorphism of $\mathscr{B}_{\theta}$ with $\mathscr{B}_{\theta \otimes \alpha}$ for every theta characteristic $\theta$. We denote by $l(\mathscr{B})$ and $l\left(\mathscr{B}_{\theta}\right)$ the length of the schemes $\mathscr{B}$ and $\mathscr{B}_{\theta}$. From the preceding we deduce the relations

$$
l(\mathscr{B})=16 \cdot l\left(\mathscr{B}_{\theta}\right) \quad \text { for every theta characteristic } \theta .
$$

\section{Grothendieck's Quot-scheme}

Let $\theta$ be a theta characteristic on $X$. We consider the functor $\underline{Q}$ from the opposite category of $k$-schemes of finite type to the category of sets defined by

$$
\begin{gathered}
\underline{\mathcal{Q}}(S)=\left\{\sigma: \pi_{X_{1}}^{*}\left(F_{*}\left(\theta^{-1}\right)\right) \rightarrow g \rightarrow 0 \mid g \text { coherent over } X_{1} \times S, \text { flat over } S,\right. \\
\left.\operatorname{deg} g_{X_{1 \times\{s\}}}=\operatorname{rk} g_{X_{1} \times\{s\}}=p-2 \text { for all } s \in S\right\} / \cong
\end{gathered}
$$

where $\pi_{X_{1}}: X_{1} \times S \rightarrow X_{1}$ denotes the natural projection and $\sigma \cong \sigma^{\prime}$ for quotients $\sigma$ and $\sigma^{\prime}$ if and only if there exists an isomorphism $\lambda: g \rightarrow g^{\prime}$ such that $\sigma^{\prime}=\lambda \circ \sigma$.

Grothendieck showed in [G] (see also [HL], Section 2.2) that the functor $\underline{Q}$ is representable by a $k$-scheme, which we denote by $\mathcal{Q}$. A $k$-point of $\mathcal{Q}$ corresponds to a quotient $\sigma: F_{*}\left(\theta^{-1}\right) \rightarrow G$, or equivalently to a rank-2 subsheaf $E=\operatorname{ker} \sigma \subset$ $F_{*}\left(\theta^{-1}\right)$ of degree 0 on $X_{1}$. By Proposition 3.1 (a) (ii) any subsheaf $E$ of degree 0 is a subbundle of $F_{*}\left(\theta^{-1}\right)$, which implies that any sheaf $g \in \underline{Q}(S)$ is locally free (see also [MuSa] or [L], Lemma 3.8). Moreover we note that by Proposition 3.1 (b) (iv) the bundle $E$ has maximal degree as a subbundle of $F_{*}\left(\theta^{-1}\right)$.

Hence taking the kernel of $\sigma$ induces a bijection of $\underline{\mathcal{Q}}(S)$ with the following set, which we also denote by $\underline{\mathcal{Q}}(S)$

$$
\begin{array}{r}
\underline{\mathcal{Q}}(S)=\left\{\mathscr{E} \hookrightarrow \pi_{X_{1}}^{*}\left(F_{*}\left(\theta^{-1}\right)\right) \mid \mathcal{E} \text { locally free sheaf over } X_{1} \times S \text { of rank } 2,\right. \\
\left.\pi_{X_{1}}^{*}\left(F_{*}\left(\theta^{-1}\right)\right) / \mathcal{E} \text { locally free, }\left.\operatorname{deg} \mathcal{E}\right|_{X_{1} \times\{s\}}=0 \text { for all } s \in S\right\} / \cong
\end{array}
$$


By Proposition 3.1 (b) the scheme $\mathcal{Q}$ decomposes as a disjoint union

$$
\mathcal{Q}=\coprod_{\eta} \mathcal{Q}_{\eta}
$$

where $\eta$ varies over the $p$-torsion points $\eta \in J X_{1}[p]_{\text {red }}=\operatorname{ker}\left(V: J X_{1} \rightarrow J X\right)$. We also denote by $V$ the Verschiebung of $J X_{1}$, i.e. $V(L)=F^{*} L$, for $L \in J X_{1}$. The set-theoretical support of $Q_{\eta}$ equals

$$
\operatorname{supp} \mathcal{Q}_{\eta}=\{E \in \operatorname{supp} \mathcal{Q} \mid \operatorname{det} E=\eta\} .
$$

Because of the projection formula, the tensor product with a $p$-torsion point $\beta \in J X_{1}[p]_{\text {red }}$ induces an isomorphism of $\mathcal{Q}_{\eta}$ with $Q_{\eta \otimes \beta}$. This implies the relation

$$
l(\mathcal{Q})=p^{2} \cdot l\left(\mathcal{Q}_{0}\right),
$$

since $X_{1}$ is assumed to be ordinary. Moreover, by Proposition 3.1 we have the settheoretical equality

$$
\operatorname{supp} \mathcal{Q}_{0}=\operatorname{supp} \mathscr{B}_{\theta}
$$

Proposition 4.1. (a) $\operatorname{dim} \mathcal{Q}=0$.

(b) The scheme $\mathcal{Q}$ is a local complete intersection at any $k$-point $e=(E \subset$ $\left.F_{*}\left(\theta^{-1}\right)\right) \in \mathcal{Q}$.

Proof. Assertion (a) follows from the preceding remarks and $\operatorname{dim} \mathscr{B}=0$. By [HL], Proposition 2.2.8, assertion (b) follows from the equality $\operatorname{dim}_{[E]} \mathcal{Q}=0=$ $\chi(\underline{\operatorname{Hom}}(E, G))$, where $E=\operatorname{ker}\left(\sigma: F_{*}\left(\theta^{-1}\right) \rightarrow G\right)$ and Hom denotes the sheaf of homomorphisms.

Let $\mathcal{N}_{X_{1}}$ denote the moduli space of semistable rank-2 vector bundles of degree 0 over $X_{1}$. We denote by $\mathcal{N}_{X_{1}}^{s}$ and $\mathcal{M}_{X_{1}}^{s}$ the open subschemes of $\mathcal{N}_{X_{1}}$ and $\mathcal{M}_{X_{1}}$ corresponding to stable vector bundles. Recall (see [La1], Theorem 4.1) that $\mathcal{N}_{X_{1}}^{s}$ and $\mathcal{M}_{X_{1}}^{s}$ universally corepresent the functors (see e.g. [HL], Definition 2.2.1) from the opposite category of $k$-schemes of finite type to the category of sets defined by

$$
\begin{aligned}
\underline{\mathcal{N}}_{X_{1}}^{s}(S)= & \left\{\mathscr{E} \text { locally free sheaf over } X_{1} \times S \text { of rank } 2|\mathcal{E}|_{X_{1} \times\{s\}}\right. \text { stable, } \\
& \left.\left.\operatorname{deg} \mathscr{E}\right|_{X_{1} \times\{s\}}=0 \text { for all } s \in S\right\} / \sim, \\
\underline{\mathcal{M}}_{X_{1}}^{s}(S)= & \left\{\mathscr{E} \text { locally free sheaf over } X_{1} \times S \text { of rank } 2|\mathcal{E}|_{X_{1} \times\{s\}}\right. \text { stable } \\
& \text { for all } \left.s \in S, \operatorname{det} \mathscr{E}=\pi_{S}^{*} M \text { for some line bundle } M \text { on } S\right\} / \sim,
\end{aligned}
$$

where $\pi_{S}: X_{1} \times S \rightarrow S$ denotes the natural projection and $\varepsilon^{\prime} \sim \varepsilon$ if and only if there exists a line bundle $L$ on $S$ such that $\mathcal{E}^{\prime} \cong \mathscr{E} \otimes \pi_{S}^{*} L$. We denote by $\langle\mathcal{E}\rangle$ the equivalence class of the vector bundle $\mathscr{E}$ for the relation $\sim$. 
Consider the determinant morphism

$$
\operatorname{det}: \mathcal{N}_{X_{1}} \rightarrow J X_{1}, \quad[E] \mapsto \operatorname{det} E,
$$

and denote by $\operatorname{det}^{-1}(0)$ the scheme-theoretical fibre over the trivial line bundle on $X_{1}$. Since $\mathcal{N}_{X_{1}}^{s}$ universally corepresents the functor $\mathcal{N}_{X_{1}}^{s}$, we have an isomorphism

$$
\mathcal{M}_{X_{1}}^{s} \cong \mathcal{N}_{X_{1}}^{s} \cap \operatorname{det}^{-1}(0) .
$$

Remark 4.2. If $p>0$, it is not known whether the canonical morphism $\mathcal{M}_{X_{1}} \rightarrow$ $\operatorname{det}^{-1}(0)$ is an isomorphism (see e.g. [La2], Section 3).

In the sequel we need the following relative version of Proposition 3.1 (b) (ii). By a $k$-scheme we always mean a $k$-scheme of finite type.

Lemma 4.3. Let $S$ be a connected $k$-scheme and let $\mathbb{E}$ be a locally free sheaf of rank-2 over $X_{1} \times S$ such that $\left.\operatorname{deg} \mathcal{E}\right|_{X_{1} \times\{s\}}=0$ for all points $s$ of $S$. Suppose that $\operatorname{Hom}\left(\mathcal{E}, \pi_{X_{1}}^{*}\left(F_{*}\left(\theta^{-1}\right)\right) \neq 0\right.$. Then we have the exact sequence

$$
0 \longrightarrow \pi_{X}^{*}(\theta) \longrightarrow\left(F \times \operatorname{id}_{S}\right)^{*} \mathscr{E} \longrightarrow \pi_{X}^{*}\left(\theta^{-1}\right) \longrightarrow 0 .
$$

In particular

$$
\left(F \times \operatorname{id}_{S}\right)^{*}(\operatorname{det} \mathcal{E})=\mathcal{O}_{X_{1} \times S} .
$$

Proof. First we note that by flat base change for $\pi_{X_{1}}: X_{1} \times S \rightarrow X_{1}$, we have an isomorphism $\pi_{X_{1}}^{*}\left(F_{*}\left(\theta^{-1}\right)\right) \cong\left(F \times \operatorname{id}_{S}\right)_{*}\left(\pi_{X}^{*}\left(\theta^{-1}\right)\right)$. Hence the nonzero morphism $\mathcal{E} \rightarrow \pi_{X_{1}}^{*}\left(F_{*}\left(\theta^{-1}\right)\right)$ gives via adjunction a nonzero morphism

$$
\varphi:\left(F \times \mathrm{id}_{S}\right)^{*} \mathcal{E} \longrightarrow \pi_{X}^{*}\left(\theta^{-1}\right) .
$$

We know by the proof of Proposition 3.1 (b) that the fibre $\varphi_{(x, s)}$ over any closed point $(x, s) \in X \times S$ is a surjective $k$-linear map. Hence $\varphi$ is surjective by Nakayama and we have an exact sequence

$$
0 \longrightarrow \mathcal{L} \longrightarrow\left(F \times \mathrm{id}_{S}\right)^{*} \mathcal{E} \longrightarrow \pi_{X}^{*}\left(\theta^{-1}\right) \longrightarrow 0,
$$

with $\mathcal{L}$ locally free sheaf of rank 1 . By $[\mathrm{K}]$, Section 5 , the rank-2 vector bundle $\left(F \times \operatorname{id}_{S}\right)^{*} \mathscr{E}$ is equipped with a canonical connection

$$
\nabla:\left(F \times \mathrm{id}_{S}\right)^{*} \mathcal{E} \longrightarrow\left(F \times \mathrm{id}_{S}\right)^{*} \mathcal{E} \otimes \Omega_{X \times S / S}^{1}
$$

We note that $\Omega_{X \times S / S}^{1}=\pi_{X}^{*}\left(\omega_{X}\right)$, where $\omega_{X}$ denotes the canonical line bundle of $X$. The first fundamental form of the connection $\nabla$ is an $\mathcal{O}_{X \times S^{-}}$-linear homomorphism

$$
\psi_{\nabla}: \mathcal{L} \longrightarrow \pi_{X}^{*}\left(\theta^{-1}\right) \otimes \pi_{X}^{*}\left(\omega_{X}\right)=\pi_{X}^{*}(\theta) .
$$


The restriction of $\psi_{\nabla}$ to the curve $X \times\{s\} \subset X \times S$ for any closed point $s \in S$ is an isomorphism (see e.g. proof of [LS], Corollary 2.6). Hence the fibre of $\psi_{\nabla}$ is a $k$-linear isomorphism over any closed point $(x, s) \in X \times S$. We conclude that $\psi_{\nabla}$ is an isomorphism, by Nakayama's lemma and because $\mathcal{L}$ is a locally free sheaf of rank 1.

We obtain the second assertion of the lemma, since

$$
\left(F \times \operatorname{id}_{S}\right)^{*}(\operatorname{det} \mathscr{E})=\operatorname{det}\left(F \times \operatorname{id}_{S}\right)^{*} \mathscr{E}=\mathcal{L} \otimes \pi_{X}^{*}\left(\theta^{-1}\right)=\mathcal{O}_{X_{1} \times S} .
$$

Proposition 4.4. We assume $X$ ordinary.

(a) The forgetful morphism

$$
i: \mathbb{Q} \hookrightarrow \mathcal{N}_{X_{1}}^{s}, \quad e=\left(E \subset F_{*}\left(\theta^{-1}\right)\right) \mapsto E
$$

is a closed embedding.

(b) The restriction $i_{0}$ of $i$ to the subscheme $\mathcal{Q}_{0} \subset \mathcal{Q}$ factors through $\mathcal{M}_{X_{1}}^{s}$, i.e. there is a closed embedding

$$
i_{0}: \mathcal{Q}_{0} \hookrightarrow \mathcal{M}_{X_{1}}^{s} .
$$

Proof. (a) Let $e=\left(E \subset F_{*}\left(\theta^{-1}\right)\right)$ be a $k$-point of $\mathcal{Q}$. To show that $i$ is a closed embedding at $e \in \mathcal{Q}$, it is enough to show that the differential $(d i)_{e}: T_{e} \mathcal{Q} \rightarrow T_{[E]} \mathcal{N}_{X_{1}}$ is injective - note that $\mathcal{Q}$ is proper. Since the bundle $E$ is stable, the Zariski tangent spaces identify with $\operatorname{Hom}(E, G)$ and $\operatorname{Ext}^{1}(E, E)$ respectively (see e.g. [HL], Proposition 2.2.7 and Corollary 4.5.2). Moreover, if we apply the functor $\operatorname{Hom}(E, \cdot)$ to the exact sequence associated with $e \in \mathbb{Q}$

$$
0 \longrightarrow E \longrightarrow F_{*}\left(\theta^{-1}\right) \longrightarrow G \longrightarrow 0,
$$

the coboundary map $\delta$ of the long exact sequence

$$
\begin{aligned}
0 \longrightarrow & \operatorname{Hom}(E, E) \longrightarrow \operatorname{Hom}\left(E, F_{*}\left(\theta^{-1}\right)\right) \\
& \longrightarrow \operatorname{Hom}(E, G) \stackrel{\delta}{\longrightarrow} \operatorname{Ext}^{1}(E, E) \longrightarrow \cdots
\end{aligned}
$$

identifies with the differential $(d i)_{e}$. By Proposition 3.1 (b) we obtain that the map $\operatorname{Hom}(E, E) \rightarrow \operatorname{Hom}\left(E, F_{*}\left(\theta^{-1}\right)\right)$ is an isomorphism. Thus $(d i)_{e}$ is injective.

(b) We consider the composite map

$$
\alpha: \mathcal{Q} \stackrel{i}{\longrightarrow} \mathcal{N}_{X_{1}}^{s} \stackrel{\operatorname{det}}{\longrightarrow} J X_{1} \stackrel{V}{\longrightarrow} J X
$$

where the last map is the isogeny given by the Verschiebung on $J X_{1}$, i.e. $V(L)=F^{*} L$ for $L \in J X_{1}$. The morphism $\alpha$ is induced by the natural transformation of functors $\underline{\alpha}: \underline{\mathcal{Q}} \Rightarrow \underline{J X}$, defined by

$$
\underline{\mathcal{Q}}(S) \longrightarrow \underline{J X}(S), \quad\left(\& \hookrightarrow \pi_{X}^{*}\left(F_{*}\left(\theta^{-1}\right)\right)\right) \mapsto\left(F \times \operatorname{id}_{S}\right)^{*}(\operatorname{det} \mathscr{E}) .
$$


Using Lemma 4.3 this immediately implies that $\alpha$ factors through the inclusion of the reduced point $\left\{\mathcal{O}_{X}\right\} \hookrightarrow J X$. Hence the image of $\mathcal{Q}$ under the composite morphism det $\circ i$ is contained in the kernel of the isogeny $V$, which is the reduced scheme $J X_{1}[p]_{\text {red }}$, since we have assumed $X$ ordinary. Taking connected components we see that the image of $\mathcal{Q}_{0}$ under det $\circ i$ is the reduced point $\left\{\mathcal{O}_{X_{1}}\right\} \hookrightarrow J X_{1}$, which implies that $i_{0}\left(\mathcal{Q}_{0}\right)$ is contained in $\mathcal{N}_{X_{1}}^{s} \cap \operatorname{det}^{-1}(0) \cong \mathcal{M}_{X_{1}}^{s}$.

In order to compare the two schemes $\mathscr{B}_{\theta}$ and $\mathcal{Q}_{0}$ we need the following lemma.

Lemma 4.5. (1) The closed subscheme $\mathscr{B} \subset \mathcal{M}_{X_{1}}^{s}$ corepresents the functor $\underline{B}$ which associates to a $k$-scheme $S$ the set

$$
\begin{aligned}
\underline{B}(S)=\{ & \mathcal{E} \text { locally free sheaf over } X_{1} \times S \text { of rank } 2|\mathcal{E}|_{X_{1} \times\{s\}} \text { stable for all } s \in S, \\
& 0 \rightarrow \mathcal{L} \rightarrow\left(F \times \operatorname{id}_{S}\right)^{*} \mathcal{E} \rightarrow \mathcal{M} \rightarrow 0 \text { for some locally free sheaves } \mathcal{L}, \mathcal{M} \\
& \text { over } X \times S \text { of rank } 1,\left.\operatorname{deg} \mathcal{L}\right|_{X \times\{s\}}=-\left.\operatorname{deg} \mathcal{M}\right|_{X \times\{s\}}=1 \text { for all } s \in S, \\
& \left.\operatorname{det} \mathscr{E}=\pi_{S}^{*} M \text { for some line bundle } M \text { on } S\right\} / \sim .
\end{aligned}
$$

(2) The closed subscheme $\mathscr{B}_{\theta} \subset \mathcal{M}_{X_{1}}^{s}$ corepresents the subfunctor $\underline{\mathcal{B}}_{\theta}$ of $\underline{\mathcal{B}}$ defined by $\langle\mathscr{E}\rangle \in \underline{B}_{\theta}(S)$ if and only if the set-theoretical image of the classifying morphism of $\mathcal{L}$

$$
\Phi_{\mathcal{L}}: S \longrightarrow \operatorname{Pic}^{1}(X),\left.\quad s \longmapsto \mathcal{L}\right|_{X \times\{s\}},
$$

is the point $\theta \in \operatorname{Pic}^{1}(X)$.

Proof. We denote by $\mathfrak{M}_{X}$ the algebraic stack parametrizing rank-2 vector bundles with trivial determinant over $X$. Let $\mathfrak{M}_{X}^{s s}$ and $\mathfrak{M}_{X}^{s}$ denote the open substacks of $\mathfrak{M}_{X}$ parametrizing semistable and stable bundles. We similarly denote the corresponding stacks of bundles over $X_{1}$. The Shatz stratification [Sh] of $\mathfrak{M}_{X}$ induced by the degree of the first piece of the Harder-Narasimhan filtration reduces in the case of rank-2 vector bundles to a filtration of the stack $\mathfrak{M}_{X}$

$$
\mathfrak{M}_{X}^{s s} \subset \mathfrak{M}_{X}^{\leq 1} \subset \mathfrak{M}_{X}^{\leq 2} \subset \cdots \subset \mathfrak{M}_{X}^{\leq n} \subset \cdots \subset \mathfrak{M}_{X}
$$

by open substacks $\mathfrak{M}_{X}^{\leq n}$. It follows from the semicontinuity of the Harder-Narasimhan filtration ([Sh], Section 5) that, for every integer $n$, there is a closed reduced substack $\mathfrak{M}_{X}^{n}$ of $\mathfrak{M}_{X}^{\leq n}$ parametrizing vector bundles having a maximal destabilizing line subbundle of degree $n$. Note that $\mathfrak{M}_{X}^{n}$ is the complement of $\mathfrak{M}_{X}^{\leq n-1}$ in $\mathfrak{M}_{X}^{\leq n}$. It can be shown (see e.g. [He], Folgerung 2.1.10) that the stacks $\mathfrak{M}_{X}^{n}$ and $\mathfrak{M}_{X}$ are smooth. Let $\mathfrak{V}: \mathfrak{M}_{X_{1}} \rightarrow \mathfrak{M}_{X}$ denote the morphism of stacks induced by pull-back under the Frobenius map $F: X \rightarrow X_{1}$. It follows from [LS], Corollary 2.6, that the restriction of $\mathfrak{V}$ to the open substack $\mathfrak{M}_{X_{1}}^{s s}$ determines a morphism of stacks

$$
\mathfrak{V}^{s s}: \mathfrak{M}_{X_{1}}^{s s} \longrightarrow \mathfrak{M}_{X}^{\leq 1}
$$


We will use the following facts about the stack $\mathfrak{M}_{X}$.

- The pull-back of $\mathcal{O}_{\mathbb{P}^{3}}(1)$ by the natural map $\mathfrak{M}_{X}^{s S} \rightarrow \mathcal{M}_{X} \cong \mathbb{P}^{3}$ extends to a line bundle, which we denote by $\mathcal{O}(1)$, over the moduli stack $\mathfrak{M}_{X}^{\leq 1}$ and $\operatorname{Pic}\left(\mathfrak{M}_{X}^{\leq 1}\right)=$ $\mathbb{Z} \cdot \mathcal{O}(1)$. Moreover, for any positive integer $l$, there is a natural isomorphism $H^{0}\left(\mathfrak{M}_{X}^{\leq 1}, \mathcal{O}(l)\right) \cong H^{0}\left(\mathcal{M}_{\mathbb{P}^{3}}, \mathcal{O}_{\mathbb{P}^{3}}(l)\right)$ (see [BL], Propositions 8.3 and 8.4).

- The closed substack $\mathfrak{M}_{X}^{1}$ is the base locus of the linear system $|\mathcal{O}(1)|$ over the stack $\mathfrak{M}_{X}^{\leq 1}$ (see Proposition A).

In order to prove part (1) it will be enough to show that the functor $\underline{\mathcal{B}}$ defined in the lemma coincides with the fibre product functor $\mathscr{B} \times \mathcal{M}_{X_{1}}^{s} \underline{\mathcal{M}}_{X_{1}}^{s}$ - we recall that $\mathcal{M}_{X_{1}}^{s}$ universally corepresents the functor $\mathcal{M}_{X_{1}}^{S}$.

We now compute the fibre product functor $\mathscr{B} \times \mathcal{M}_{X_{1}}^{s} \mathcal{M}_{X_{1}}^{s}$. Let $S$ be a $k$-scheme and consider a vector bundle $\mathscr{E} \in \mathfrak{M}_{X_{1}}^{s}(S)$. Since the subscheme $\mathscr{B}$ is defined as the base locus of the linear system $V^{*}\left|\mathcal{O}_{\mathbb{P}^{3}}(1)\right|$, we obtain that $\langle\mathscr{E}\rangle \in\left[\mathcal{B} \times \mathcal{M}_{X_{1}}^{s} \underline{\mathcal{M}}_{X_{1}}^{s}\right](S)$ if and only if $\mathscr{E}$ lies in the base locus of $\mathfrak{V}^{s S *}|\mathcal{O}(1)|$ - here we use the isomorphism $\left|\mathcal{O}_{\mathbb{P}^{3}}(1)\right| \cong|\mathcal{O}(1)|$, or equivalently $\mathfrak{V}^{s s}(\mathcal{E}):=\left(F \times \mathrm{id}_{S}\right)^{*} \mathscr{E} \in \mathfrak{M}_{X}^{\leq 1}(S)$ lies in the base locus of $|\mathcal{O}(1)|$, which is the closed substack $\mathfrak{M}_{X}^{1}$.

We now consider the universal exact sequence defined by the Harder-Narasimhan filtration over $\mathfrak{M}_{X}^{1}$ :

$$
0 \rightarrow \mathcal{L} \rightarrow\left(F \times \mathrm{id}_{S}\right)^{*} \mathcal{E} \rightarrow \mathcal{M} \rightarrow 0,
$$

with $\mathcal{L}, \mathcal{M}$ locally free sheaves over $X \times S$ such that $\left.\operatorname{deg} \mathcal{L}\right|_{X \times\{s\}}=-\left.\operatorname{deg} \mathcal{M}\right|_{X \times\{s\}}=1$ for any $s \in S$. This shows that the two sets $\left[\mathcal{B} \times{ }_{\mathcal{M}_{X_{1}}^{s}} \underline{\mathcal{M}}_{X_{1}}^{s}\right](S)$ and $\underline{B}(S)$ coincide. This proves (1).

As for (2), we add the condition that the family $\mathcal{E}$ is Frobenius-destabilized by the theta-characteristic $\theta$.

Remark 4.6. Note that in Lemma 4.5 we do not need to assume $X$ ordinary.

Proposition 4.7. We assume $X$ ordinary. There is a scheme-theoretical equality

$$
\mathcal{B}_{\theta}=Q_{0}
$$

as closed subschemes of $\mathcal{M}_{X_{1}}$.

Proof. Since $\mathcal{B}_{\theta}$ and $\mathcal{Q}_{0}$ corepresent the two functors $\underline{\mathcal{B}}_{\theta}$ and $\underline{\mathcal{Q}}_{0}$ it will be enough to show that there is a canonical bijection between the set $\underline{\mathcal{B}}_{\theta}(S)$ and $\underline{\mathcal{Q}}_{0}(S)$ for any $k$-scheme $S$. We recall that

$$
\begin{aligned}
& \underline{\mathcal{Q}}_{0}(S)=\left\{\mathscr{E} \hookrightarrow \pi_{X_{1}}^{*}\left(F_{*}\left(\theta^{-1}\right)\right) \mid \mathcal{E} \text { locally free sheaf over } X_{1} \times S \text { of rank } 2,\right. \\
&\left.\pi_{X}^{*}\left(F_{*}\left(\theta^{-1}\right)\right) / \mathscr{E} \text { locally free, det } \mathscr{E} \cong \mathcal{O}_{X_{1} \times S}\right\} / \cong
\end{aligned}
$$


Note that the property det $\mathscr{E} \cong \mathcal{O}_{X_{1} \times S}$ is implied as follows: by Proposition 4.4 (b) we have det $\mathscr{E} \cong \pi_{S}^{*} L$ for some line bundle $L$ over $S$ and by Lemma 4.3 we conclude that $L=\mathcal{O}_{S}$.

First we show that the natural map $\underline{\mathcal{Q}}_{0}(S) \longrightarrow \underline{\mathcal{M}}_{X_{1}}^{S}(S)$ is injective. Suppose that there exist $\mathscr{E}, \mathscr{E}^{\prime} \in \underline{\mathcal{Q}}_{0}(S)$ such that $\langle\mathscr{E}\rangle=\left\langle\mathcal{E}^{\prime}\right\rangle$, i.e. $\mathcal{E}^{\prime} \cong \mathscr{E} \otimes \pi_{S}^{*}(L)$ for some line bundle $L$ on $S$. Then by Lemma 4.3 we have two inclusions

$$
i: \pi_{X}^{*}(\theta) \longrightarrow\left(F \times \mathrm{id}_{S}\right)^{*} \mathcal{E}, \quad i^{\prime}: \pi_{X}^{*}(\theta) \otimes \pi_{S}^{*}\left(L^{-1}\right) \longrightarrow\left(F \times \mathrm{id}_{S}\right)^{*} \mathcal{E} .
$$

Composing with the projection $\sigma:\left(F \times \mathrm{id}_{S}\right)^{*} \mathcal{E} \rightarrow \pi_{X}^{*}\left(\theta^{-1}\right)$ we see that the composite map $\sigma \circ i^{\prime}$ is identically zero. Hence the two subbundles $\pi_{X}^{*}(\theta)$ and $\pi_{X}^{*}(\theta) \otimes \pi_{S}^{*}\left(L^{-1}\right)$ coincide, which implies $\pi_{S}^{*}(L)=\mathcal{O}_{X_{1} \times S}$.

Therefore the two sets $\underline{\mathcal{Q}}_{0}(S)$ and $\underline{\mathcal{B}}_{\theta}(S)$ are naturally subsets of $\underline{\mathcal{M}}_{X_{1}}^{S}(S)$.

We now show that $\underline{\underline{Q}}_{0}(S) \subset \underline{\mathcal{B}}_{\theta}(S)$. Consider $\& \in \underline{\mathscr{Q}}_{0}(S)$. By Proposition 3.1 (b) the bundle $\left.\mathscr{E}\right|_{X_{1} \times\{s\}}$ is stable for all $s \in S$. By Lemma 4.3 we can take $\mathcal{L}=\pi_{X}^{*}(\theta)$ and $\mathcal{M}=\pi_{X}^{*}\left(\theta^{-1}\right)$, so that $\langle\mathscr{E}\rangle \in \underline{\mathcal{B}}_{\theta}(S)$.

Hence it remains to show that $\underline{\mathcal{B}}_{\theta}(S) \subset \underline{\mathcal{Q}}_{0}(S)$. Consider a sheaf $\mathbb{E}$ with $\langle\mathscr{E}\rangle \in \mathbb{B}_{\theta}(S)$ - see Lemma 4.5 (2). As in the proof of Lemma 4.3 we consider the canonical connection $\nabla$ on $\left(F \times \mathrm{id}_{S}\right)^{*} \mathcal{E}$. Its first fundamental form is an $\mathcal{O}_{X \times S^{-}}$ linear homomorphism

$$
\psi_{\nabla}: \mathcal{L} \longrightarrow \mathcal{M} \otimes \pi_{X}^{*}\left(\omega_{X}\right),
$$

which is surjective on closed points $(x, s) \in X \times S$. Hence we can conclude that $\psi_{\nabla}$ is an isomorphism. Moreover taking the determinant, we obtain

$$
\mathcal{L} \otimes \mathcal{M}=\operatorname{det}\left(F \times \mathrm{id}_{S}\right)^{*} \mathcal{E}=\pi_{S}^{*} M,
$$

for some line bundle $M$ on $S$. Combining both isomorphisms we deduce that

$$
\mathcal{L} \otimes \mathcal{L}=\pi_{X}^{*}\left(\omega_{X}\right) \otimes \pi_{S}^{*} M .
$$

Hence its classifying morphism $\Phi_{\mathscr{L} \otimes \mathscr{L}}: S \longrightarrow \operatorname{Pic}^{2}(X)$ factorizes through the inclusion of the reduced point $\left\{\omega_{X}\right\} \hookrightarrow \operatorname{Pic}^{2}(X)$. Moreover the composite map of $\Phi_{\mathscr{L}}$ with the duplication map [2]

$$
\Phi_{\mathscr{L} \otimes \mathcal{L}}: S \stackrel{\Phi_{\mathcal{L}}}{\longrightarrow} \operatorname{Pic}^{1}(X) \stackrel{[2]}{\longrightarrow} \operatorname{Pic}^{2}(X)
$$

coincides with $\Phi_{\mathscr{L} \otimes \mathcal{L}}$. We deduce that $\Phi_{\mathscr{L}}$ factorizes through the inclusion of the reduced point $\{\theta\} \hookrightarrow \operatorname{Pic}^{1}(X)$. Note that the fibre $[2]^{-1}\left(\omega_{X}\right)$ is reduced, since $p>2$. Since $\operatorname{Pic}^{1}(X)$ is a fine moduli space, there exists a line bundle $N$ over $S$ such that

$$
\mathcal{L}=\pi_{X}^{*}(\theta) \otimes \pi_{S}^{*}(N) .
$$


We introduce the vector bundle $\varepsilon_{0}=\mathscr{E} \otimes \pi_{S}^{*}\left(N^{-1}\right)$. Then $\left\langle\varepsilon_{0}\right\rangle=\langle\mathscr{E}\rangle$ and we have an exact sequence

$$
0 \longrightarrow \pi_{X}^{*}(\theta) \longrightarrow\left(F \times \mathrm{id}_{S}\right)^{*} \varepsilon_{0} \stackrel{\sigma}{\longrightarrow} \pi_{X}^{*}\left(\theta^{-1}\right) \longrightarrow 0,
$$

since $\pi_{S}^{*} M=\pi_{S}^{*} N^{2}$. By adjunction the morphism $\sigma$ gives a nonzero morphism

$$
j: \varepsilon_{0} \longrightarrow\left(F \times \operatorname{id}_{S}\right)_{*}\left(\pi_{X}^{*}\left(\theta^{-1}\right)\right) \cong \pi_{X_{1}}^{*}\left(F_{*}\left(\theta^{-1}\right)\right) .
$$

We now show that $j$ is injective. Suppose it is not. Then there exists a subsheaf $\tilde{\mathcal{E}}_{0} \subset \pi_{X_{1}}^{*}\left(F_{*}\left(\theta^{-1}\right)\right)$ and a surjective map $\tau: \varepsilon_{0} \rightarrow \tilde{\varepsilon}_{0}$. Let $\mathcal{K}$ denote the kernel of $\tau$. Again by adjunction we obtain a map $\alpha:\left(F \times \operatorname{id}_{S}\right)^{*} \tilde{\varepsilon}_{0} \rightarrow \pi_{X}^{*}\left(\theta^{-1}\right)$ such that the composite map

$$
\left(F \times \operatorname{id}_{S}\right)^{*} \varepsilon_{0} \stackrel{\tau^{*}}{\longrightarrow}\left(F \times \operatorname{id}_{S}\right)^{*} \tilde{\mathcal{E}}_{0} \stackrel{\alpha}{\longrightarrow} \pi_{X}^{*}\left(\theta^{-1}\right)
$$

coincides with $\sigma$. Here $\tau^{*}$ denotes the map $\left(F \times \mathrm{id}_{S}\right)^{*} \tau$. Since $\sigma$ is surjective, $\alpha$ is also surjective. We denote by $\mathcal{M}$ the kernel of $\alpha$. The induced map $\bar{\tau}: \pi_{X}^{*}(\theta)=\operatorname{ker} \sigma \rightarrow$ $\mathcal{M}$ is surjective, because $\tau^{*}$ is surjective. Moreover the first fundamental form of the canonical connection $\tilde{\nabla}$ on $\left(F \times \mathrm{id}_{S}\right)^{*} \tilde{\varepsilon}_{0}$ induces an $\mathcal{O}_{X \times S}$-linear homomorphism $\psi_{\tilde{\nabla}}: \mathcal{M} \rightarrow \pi_{X}^{*}(\theta)$ and the composite map

$$
\psi_{\nabla}: \pi_{X}^{*}(\theta) \stackrel{\bar{\tau}}{\longrightarrow} \mathcal{M} \stackrel{\psi_{\tilde{\nabla}}}{\longrightarrow} \pi_{X}^{*}(\theta)
$$

coincides with the first fundamental form of $\nabla$ of $\left(F \times \mathrm{id}_{S}\right)^{*} \varepsilon_{0}$, which is an isomorphism. Therefore $\bar{\tau}$ is an isomorphism too. So $\tau^{*}$ is an isomorphism and $\left(F \times \operatorname{id}_{S}\right)^{*} \mathcal{K}=0$. We deduce that $\mathcal{K}=0$.

In order to show that $\varepsilon_{0} \in \underline{Q}_{0}(S)$, it remains to verify that the quotient sheaf $\pi_{X_{1}}\left(F_{*}\left(\theta^{-1}\right)\right) / \mathcal{E}_{0}$ is flat over $S$. We recall that flatness implies locally freeness because of maximality of degree. But flatness follows from [HL], Lemma 2.1.4, since the restriction of $j$ to $X_{1} \times\{s\}$ is injective for any closed $s \in S$ by Proposition 3.1 (a).

Since $\mathcal{Q}_{0}$ represents the functor $\underline{\mathcal{Q}}_{0}$, we obtain the following

Corollary 4.8. The scheme $\mathscr{B}_{\theta}$ represents the functor $\underline{\mathcal{B}}_{\theta}$ defined in Lemma 4.5.

Combining Proposition 4.7 with relations (7) and (8), we obtain

Corollary 4.9. We have

$$
l(\mathscr{B})=\frac{16}{p^{2}} \cdot l(\mathcal{Q})
$$




\section{Determinantal subschemes}

In this section we introduce a determinantal subscheme $\mathscr{D} \subset \mathcal{N}_{X_{1}}$, whose length will be computed in the next section. We also show that $\mathscr{D}$ is isomorphic to Grothendieck's Quot-scheme $\mathcal{Q}$. We first define a determinantal subscheme $\tilde{D}$ of a variety $J X_{1} \times Z$ covering $\mathcal{N}_{X_{1}}$ and then we show that $\tilde{D}$ is a $\mathbb{P}^{1}$-fibration over an étale cover of $\mathscr{D} \subset \mathcal{N}_{X_{1}}$.

Since there does not exist a universal bundle over $X_{1} \times \mathcal{M}_{X_{1}}$, following an idea of Mukai [Mu], we consider the moduli space $\mathcal{M}_{X_{1}}(x)$ of stable rank-2 vector bundles on $X_{1}$ with determinant $\mathcal{O}_{X_{1}}(x)$ for a fixed point $x \in X_{1}$. According to [N1] the variety $\mathcal{M}_{X_{1}}(x)$ is a smooth intersection of two quadrics in $\mathbb{P}^{5}$. Let $\mathcal{U}$ denote a universal bundle on $X_{1} \times \mathcal{M}_{X_{1}}(x)$ and denote

$$
U_{x}:=\left.U\right|_{\{x\} \times \mathcal{M}_{X_{1}}(x)}
$$

considered as a rank-2 vector bundle on $\mathcal{M}_{X_{1}}(x)$. Then the projectivized bundle

$$
Z:=\mathbb{P}\left(U_{x}\right)
$$

is a $\mathbb{P}^{1}$-bundle over $\mathcal{M}_{X_{1}}(x)$. The variety $Z$ parametrizes pairs $\left(F_{z}, l_{z}\right)$ consisting of a stable vector bundle $F_{z} \in \mathcal{M}_{X_{1}}(x)$ and a non-trivial linear form $l_{z}: F_{z}(x) \rightarrow k_{x}$ on the fibre of $F_{z}$ over $x$ defined up to a non-zero constant. Thus to any $z \in Z$ one can associate an exact sequence

$$
0 \rightarrow E_{z} \rightarrow F_{z} \rightarrow k_{x} \rightarrow 0
$$

uniquely determined up to a multiplicative constant. Clearly $E_{z}$ is semistable, since $F_{z}$ is stable, and $\operatorname{det} E_{z}=\mathcal{O}_{X_{1}}$. Hence we get a diagram (the so-called Hecke correspondence)

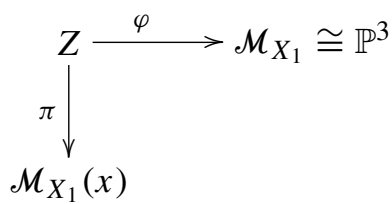

with $\varphi(z)=\left[E_{z}\right]$ and $\pi(z)=F_{z}$. We note that there is an isomorphism $\varphi^{-1}(E) \cong \mathbb{P}^{1}$ (see e.g. [Mu], (3.7)) and that $\pi\left(\varphi^{-1}(E)\right) \subset \mathcal{M}_{X_{1}}(x) \subset \mathbb{P}^{5}$ is a conic for any stable $E \in \mathcal{M}_{X_{1}}^{s}$ (see e.g. [NR2]). On $X_{1} \times Z$ there exists a "universal" bundle, which we denote by $\mathcal{V}$ (see $[\mathrm{Mu}],(3.8))$. It has the property

$$
\left.\mathcal{V}\right|_{X_{1} \times\{z\}} \cong E_{z}, \quad \text { for all } z \in Z .
$$

Let $\mathcal{L}$ denote a Poincaré bundle on $X_{1} \times J X_{1}$. By abuse of notation we also denote by $\mathcal{V}$ and $\mathcal{L}$ their pull-backs to $X_{1} \times J X_{1} \times Z$. We denote by $\pi_{X_{1}}$ and $q$ the 
canonical projections

$$
X_{1} \stackrel{\pi_{X_{1}}}{\longleftarrow} X_{1} \times J X_{1} \times Z \stackrel{q}{\longrightarrow} J X_{1} \times Z
$$

We consider the map $m$ given by tensor product

$$
m: J X_{1} \times \mathcal{M}_{X_{1}} \longrightarrow \mathcal{N}_{X_{1}}, \quad(L, E) \longmapsto L \otimes E
$$

Note that the restriction of $m$ to the stable locus $m^{s}: J X_{1} \times \mathcal{M}_{X_{1}}^{s} \longrightarrow \mathcal{N}_{X_{1}}^{s}$ is an étale map of degree 16 . We denote by $\psi$ the composite map

$$
\psi: J X_{1} \times Z \stackrel{\operatorname{id}_{J X_{1}} \times \varphi}{\longrightarrow} J X_{1} \times \mathcal{M}_{X_{1}} \stackrel{m}{\longrightarrow} \mathcal{N}_{X_{1}}, \quad \psi(L, z)=L \otimes E_{z} .
$$

Let $D \in\left|\omega_{X_{1}}\right|$ be a smooth canonical divisor on $X_{1}$. We introduce the following sheaves over $J X_{1} \times Z$

and

$$
\mathcal{F}_{1}=q_{*}\left(\mathcal{L}^{*} \otimes \mathcal{V}^{*} \otimes \pi_{X_{1}}^{*}\left(F_{*}\left(\theta^{-1}\right) \otimes \omega_{X_{1}}\right)\right)
$$

$$
\widetilde{F}_{0}=\oplus_{y \in D}\left(\left.\mathcal{L}^{*} \otimes \mathcal{V}^{*}\right|_{\{y\} \times J X_{1} \times Z}\right) \otimes k^{\oplus p} .
$$

The next proposition is an even degree analogue of [LN], Theorem 3.1.

Proposition 5.1. (a) The sheaves $\mathcal{F}_{0}$ and $\mathscr{F}_{1}$ are locally free of rank $4 p$ and $4 p-4$ respectively and there is an exact sequence

$$
0 \longrightarrow \mathcal{F}_{1} \stackrel{\gamma}{\longrightarrow} \mathcal{F}_{0} \longrightarrow R^{1} q_{*}\left(\mathcal{L}^{*} \otimes \mathcal{V}^{*} \otimes \pi_{X_{1}}^{*}\left(F_{*}\left(\theta^{-1}\right)\right)\right) \longrightarrow 0 .
$$

Let $\tilde{D} \subset J X_{1} \times Z$ denote the subscheme defined by the 4-th Fitting ideal of the sheaf $R^{1} q_{*}\left(\mathscr{L}^{*} \otimes \mathcal{V}^{*} \otimes \pi_{X_{1}}^{*}\left(F_{*}\left(\theta^{-1}\right)\right)\right)$. We have set-theoretically

$$
\operatorname{supp} \tilde{D}=\left\{(L, z) \in J X_{1} \times Z \mid \operatorname{dim} \operatorname{Hom}\left(L \otimes E_{z}, F_{*}\left(\theta^{-1}\right)\right)=1\right\},
$$

and $\operatorname{dim} \tilde{D}=1$.

(b) Let $\delta$ denote the $l$-adic $(l \neq p)$ cohomology class of $\tilde{D}$ in $J X_{1} \times Z$. Then

$$
\delta=c_{5}\left(\mathcal{F}_{0}-\mathcal{F}_{1}\right) \in H^{10}\left(J X_{1} \times Z, \mathbb{Z}_{l}\right) .
$$

Proof. We consider the canonical exact sequence over $X_{1} \times J X_{1} \times Z$ associated to the effective divisor $\pi_{X_{1}}^{*} D$

$$
\begin{aligned}
0 \rightarrow \mathcal{L}^{*} \otimes \mathcal{V}^{*} \otimes \pi_{X_{1}}^{*} F_{*}\left(\theta^{-1}\right) \stackrel{\otimes D}{\longrightarrow} \mathcal{L}^{*} \otimes \mathcal{V}^{*} \otimes \pi_{X_{1}}^{*}\left(F_{*}\left(\theta^{-1}\right) \otimes \omega_{X_{1}}\right) \\
\left.\quad \rightarrow \mathcal{L}^{*} \otimes \mathcal{V}^{*}\right|_{\pi_{X_{1}}^{*} D} \otimes k^{\oplus p} \rightarrow 0 .
\end{aligned}
$$


By Proposition 1.2 the rank- $p$ vector bundle $F_{*}\left(\theta^{-1}\right)$ is stable and since

$$
1-\frac{2}{p}=\mu\left(F_{*}\left(\theta^{-1}\right)\right)>\mu(L \otimes E)=0 \quad \text { for all }(L, E) \in J X_{1} \times \mathcal{M}_{X_{1}},
$$

we obtain

$$
\operatorname{dim} H^{1}\left(L^{*} \otimes E^{*} \otimes F_{*}\left(\theta^{-1}\right) \otimes \omega_{X_{1}}\right)=\operatorname{dim} \operatorname{Hom}\left(F_{*}\left(\theta^{-1}\right), L \otimes E\right)=0 .
$$

This implies

$$
R^{1} q_{*}\left(\mathcal{L}^{*} \otimes \mathcal{V}^{*} \otimes \pi_{X_{1}}^{*}\left(F_{*}\left(\theta^{-1}\right) \otimes \omega_{X_{1}}\right)\right)=0 .
$$

By the base change theorems the sheaf $\mathcal{F}_{1}$ is locally free. Taking direct images by $q$ (note that $q_{*}\left(\mathcal{L}^{*} \otimes \mathcal{V}^{*} \otimes \pi_{X_{1}}^{*} F_{*}\left(\theta^{-1}\right)\right)=0$ because it is a torsion sheaf), we obtain the exact sequence

$$
0 \longrightarrow \mathcal{F}_{1} \stackrel{\gamma}{\longrightarrow} \mathcal{F}_{0} \longrightarrow R^{1} q_{*}\left(\mathcal{L}^{*} \otimes \mathcal{V}^{*} \otimes \pi_{X_{1}}^{*}\left(F_{*}\left(\theta^{-1}\right)\right)\right) \longrightarrow 0 .
$$

with $\mathcal{F}_{1}$ and $\mathcal{F}_{0}$ as in the statement of the proposition. Note that by Riemann-Roch we have

$$
\text { rk } \mathcal{F}_{1}=4 p-4 \quad \text { and } \quad \operatorname{rk} \mathcal{F}_{0}=4 p .
$$

It follows from the proof of Proposition 3.1 (a) that any nonzero homomorphism $L \otimes E \longrightarrow F_{*}\left(\theta^{-1}\right)$ is injective. Moreover by Proposition 3.1 (b) (iii) for any subbundle $L \otimes E \subset F_{*}\left(\theta^{-1}\right)$ we have $\operatorname{dim} \operatorname{Hom}\left(L \otimes E, F_{*}\left(\theta^{-1}\right)\right)=1$, or equivalently $\operatorname{dim} H^{1}\left(L^{*} \otimes E^{*} \otimes F_{*}\left(\theta^{-1}\right)\right)=5$. Using the base change theorems we obtain the following series of equivalences

$$
\begin{aligned}
(L, z) \in \operatorname{supp} \tilde{D} & \Longleftrightarrow \operatorname{rk} \gamma_{(L, z)}<4 p-4=\mathrm{rk} \mathcal{F}_{1} \\
& \Longleftrightarrow \operatorname{dim} H^{1}\left(L^{*} \otimes E^{*} \otimes F_{*}\left(\theta^{-1}\right)\right) \geq 5 \\
& \Longleftrightarrow \operatorname{dim} \operatorname{Hom}\left(L \otimes E, F_{*}\left(\theta^{-1}\right)\right) \geq 1 \\
& \Longleftrightarrow \operatorname{dim} \operatorname{Hom}\left(L \otimes E, F_{*}\left(\theta^{-1}\right)\right)=1 .
\end{aligned}
$$

Finally we clearly have the equality $\operatorname{supp} \psi(\tilde{D})=\operatorname{supp} \mathcal{Q}$. Since $\operatorname{dim} \mathcal{Q}=0$ and since $\varphi^{-1}(E) \cong \mathbb{P}^{1}$ for $E$ stable, we deduce that $\operatorname{dim} \tilde{D}=1$. This proves part (a).

Part (b) follows from Porteous' formula, which says that the fundamental class $\delta \in H^{10}\left(J X_{1} \times Z, \mathbb{Z}_{l}\right)$ of the determinantal subscheme $\tilde{D}$ is given (with the notation of $[\mathrm{ACGH}]$, p. 86) by

$$
\begin{aligned}
\delta & =\Delta_{4 p-(4 p-5), 4 p-4-(4 p-5)}\left(c_{t}\left(\mathcal{F}_{0}-\mathcal{F}_{1}\right)\right) \\
& =\Delta_{5,1}\left(c_{t}\left(\mathcal{F}_{0}-\mathcal{F}_{1}\right)\right) \\
& =c_{5}\left(\widetilde{F}_{0}-\mathcal{F}_{1}\right) .
\end{aligned}
$$


Let $M$ be a sheaf over a $k$-scheme $S$. We denote by

$$
\operatorname{Fitt}_{n}[M] \subset \mathcal{O}_{S}
$$

the $n$-th Fitting ideal sheaf of $M$.

We now define the 0 -dimensional subscheme $\mathscr{D} \subset \mathcal{N}_{X_{1}}^{s}$, which is supported on $\operatorname{supp} \mathcal{Q}$, by defining a scheme structure $\mathscr{D}_{E}$ for every $E \in \operatorname{supp} \mathcal{Q}$. Note that

$$
\mathscr{D}=\coprod_{E \in \operatorname{supp} \mathcal{Q}} \mathscr{D}_{E}
$$

Consider a bundle $E \in \mathcal{N}_{X_{1}}^{s}$ with $E \in \operatorname{supp} \mathcal{Q}$, i.e.

$$
\operatorname{dim} \operatorname{Hom}\left(E, F_{*}\left(\theta^{-1}\right)\right) \geq 1 \Longleftrightarrow \operatorname{dim} H^{1}\left(E^{*} \otimes F_{*}\left(\theta^{-1}\right)\right) \geq 5 .
$$

The GIT-construction of the moduli space $\mathcal{N}_{X_{1}}^{s}$ realizes $\mathcal{N}_{X_{1}}^{s}$ as a quotient of an open subset $\mathcal{U}$ of a Quot-scheme by the group $\mathbb{P} \operatorname{GL}(N)$ for some $N$. It can be shown (see e.g. [La2], Section 3) that $U$ is a principal $\mathbb{P} G L(N)$-bundle for the étale topology over $\mathcal{N}_{X_{1}}^{s}$. Hence there exists an étale neighbourhood $\tau: \bar{U} \rightarrow U$ of $E$ over which the $\mathbb{P} G L(N)$-bundle is trivial, i.e., admits a section. The universal bundle over the Quot-scheme restricts to a bundle $\&$ over $X_{1} \times \bar{U}$. Choose a point $\bar{E} \in \bar{U}$ over $E$. We denote by $\mathscr{D}_{\bar{E}}$ the connected component supported at $\bar{E}$ of the scheme defined by the Fitting ideal sheaf

$$
\operatorname{Fitt}_{4}\left[R^{1} \pi_{\bar{U} *}\left(\mathcal{E}^{*} \otimes \pi_{X_{1}}^{*} F_{*}\left(\theta^{-1}\right)\right)\right] .
$$

Lemma 5.2. Let $\tau: \bar{U} \rightarrow U$ be an étale map and $y \in \bar{U}, x \in U$ such that $\tau(y)=x$. Let $\bar{\Lambda} \subset \bar{U}$ be a 0 -dimensional scheme supported at $y$. Then the restriction of $\tau$ to $\bar{\Lambda}$ induces an isomorphism of $\bar{\Lambda}$ with its scheme-theoretical image in $\Delta=\tau(\bar{\Lambda}) \subset U$, i.e.

$$
\left.\tau\right|_{\bar{\Lambda}}: \bar{\Lambda} \stackrel{\sim}{\longrightarrow} \Delta \subset U
$$

Proof. We denote by $A=\mathcal{O}_{\bar{U}, y}, B=\mathcal{O}_{U, x}$ the local rings at the points $y, x$ and by $\mathfrak{m}_{A}, \mathfrak{m}_{B}$ their maximal ideals. Let $I \subset \mathfrak{m}_{A}$ denote the ideal defining the scheme $\bar{\Lambda}$. Since $\operatorname{dim} \bar{\Lambda}=0$ there exists an integer $n$ such that $\mathfrak{m}_{A}^{n} \subset I$. The natural map $B \hookrightarrow A \rightarrow A / I$ factorizes as follows

$$
\beta: B \rightarrow B / \mathfrak{m}_{B}^{n} \stackrel{\alpha}{\longrightarrow} A / \mathfrak{m}_{A}^{n} \rightarrow A / I .
$$

Note that $\alpha$ is an isomorphism, since $\tau$ is étale (see e.g. [Mum], Corollary 1 of Theorem III.5.3). This shows that $\beta$ is surjective, hence $\left.\tau\right|_{\bar{\Lambda}}$ is an isomorphism. 
Proposition-Definition 5.3. For $E \in \operatorname{supp} Q 2$ we define $\mathscr{D}_{E}$ as the scheme-theoretical image $\tau\left(D_{\bar{E}}\right) \subset \mathcal{N}_{X_{1}}^{s}$ under the étale map $\tau$. Then the scheme $\mathscr{D}_{E}$ does not depend on the étale neighbourhood $\tau: \bar{U} \rightarrow U$ of $E$ and the point $\bar{E}$.

Proof. Consider for $i=1,2$ étale neighbourhoods $\tau_{i}: \bar{U}_{i} \rightarrow U$ such that universal bundles $\mathcal{E}_{i}$ exist over $X_{1} \times \bar{U}_{i}$, and points $\bar{E}_{i} \in \bar{U}_{i}$ lying over $E \in U$. Because of Lemma 5.2 it will be enough to show that the schemes $\mathscr{D}_{\bar{E}_{1}}$ and $\mathscr{D}_{\bar{E}_{2}}$ are isomorphic.

Consider the fibre product $\bar{U}=\bar{U}_{1} \times_{U} \bar{U}_{2}$ and the point $\bar{E}=\left(\bar{E}_{1}, \bar{E}_{2}\right) \in \bar{U}$. The two projections $\pi_{i}: \bar{U} \rightarrow \bar{U}_{i}$ for $i=1,2$ are étale. Moreover $\left(\operatorname{id}_{X_{1}} \times \pi_{i}\right)^{*} \mathcal{E}_{i} \sim \mathcal{E}$, where $\&$ denotes the universal bundle over $X_{1} \times \bar{U}$. Since the formation of the Fitting ideal and taking the higher direct image $R^{1} \pi_{\bar{U} *}$ commutes with the flat base changes $\pi_{1}$ and $\pi_{2}$ (see [E], Corollary 20.5), we obtain for $i=1,2$

$$
\pi_{i}^{-1}\left[\operatorname{Fitt}_{4}\left(R^{1} \pi_{\bar{U}_{1 *}}\left(\mathcal{E}_{i}^{*} \otimes \pi_{X_{1}}^{*} F_{*}\left(\theta^{-1}\right)\right)\right] \cdot \mathcal{O}_{\bar{U}}=\operatorname{Fitt}_{4}\left(R^{1} \pi_{\bar{U}_{*}}\left(\mathcal{E}^{*} \otimes \pi_{X_{1}}^{*} F_{*}\left(\theta^{-1}\right)\right)\right) .\right.
$$

This shows that the connected components supported at $\bar{E}$ of the fibres $\pi_{i}^{-1}\left(\mathscr{D}_{\bar{E}_{i}}\right)$ equal $\mathscr{D}_{\bar{E}}$. Applying Lemma 5.2 to $\pi_{i}$ and $\mathscr{D}_{\bar{E}}$ we obtain isomorphisms $\pi_{i}: \mathscr{D}_{\bar{E}} \rightarrow$ $\mathscr{D}_{\bar{E}_{i}}$ and we are done.

Lemma 5.4. (a) Let $S$ be a $k$-scheme and $\mathscr{E}$ a sheaf over $X_{1} \times S$ with $\langle\mathcal{E}\rangle \in \mathcal{N}_{X_{1}}^{s}(S)$. We suppose that the set-theoretical image of the classifying morphism of $\mathcal{E}$

$$
\Phi_{\mathcal{E}}: S \longrightarrow \mathcal{N}_{X_{1}}^{s},\left.\quad s \longmapsto \mathcal{E}\right|_{X_{1} \times\{s\}}
$$

is a point. Then there exists an Artinian ring $A$, a morphism $\varphi: S \longrightarrow \Delta:=\operatorname{Spec}(A)$ and a locally free sheaf $\varepsilon_{0}$ over $X_{1} \times \Delta$ such that

(1) $\mathcal{E} \sim\left(\operatorname{id}_{X_{1}} \times \varphi\right)^{*} \varepsilon_{0}$

(2) the natural map $\mathcal{O}_{\Delta} \longrightarrow \varphi_{*} \mathcal{O}_{S}$ is injective.

(b) There exists a universal family $\varepsilon_{0}$ over $X_{1} \times \mathbb{D}$.

Proof. (a) Since the set-theoretical support of im $\Phi_{\mathcal{E}}$ is a point $x \in \mathcal{N}_{X_{1}}^{s}$, there exists an Artinian ring $A$ such that $\Phi_{\mathcal{E}}$ factorizes through the inclusion $\Delta:=\operatorname{Spec}(A) \hookrightarrow$ $\mathcal{N}_{X_{1}}^{s}$. As explained above there exists an étale neighbourhood $\tau: \bar{U} \rightarrow U$ of $x$ such that there is a universal bundle $\mathcal{E}^{\mathrm{univ}}$ over $X_{1} \times \bar{U}$. Choose $y \in \bar{U}$ such that $\tau(y)=x$ and denote by $\bar{\Lambda} \subset \bar{U}$ the connected component supported at $y$ of the fibre $\tau^{-1}(\Delta)$. By Lemma 5.2 there is an isomorphism $\tau: \bar{\Lambda} \stackrel{\sim}{\longrightarrow} \Delta$. Denote by $\mathcal{E}_{0}$ the restriction of $\mathcal{E}^{\text {univ }}$ to $X_{1} \times \bar{\Lambda} \cong X_{1} \times \Delta$. This shows property (1). As for (2), we consider the ideal $I \subset A$ defined by $\tilde{I}=\operatorname{ker}\left(\mathcal{O}_{\operatorname{Spec}(A)} \rightarrow \varphi_{*} \mathcal{O}_{S}\right)$, where $\tilde{I}$ denotes the associated $\mathcal{O}_{\mathrm{Spec}(A)}$-module. If $I \neq 0$, we replace $A$ by $A / I$ and we are done.

(b) We take $\Delta=\mathscr{D}_{E}$ and $\bar{\Lambda}=\mathscr{D}_{\bar{E}}$ and proceed as in (a). 
Proposition 5.5. The subscheme $\mathscr{D} \subset \mathcal{N}_{X_{1}}^{s}$ represents the functor $\underline{D}$ which associates to any $k$-scheme $S$ the set

$$
\begin{gathered}
\underline{D}(S)=\left\{\text { E locally free sheaf over } X_{1} \times S \text { of rank } 2|\operatorname{deg} \mathscr{E}|_{X_{1} \times\{s\}}=0\right. \\
\text { for all } s \in S, \operatorname{Fitt}_{4}\left[R^{1} \pi_{S *}\left(\mathcal{E}^{*} \otimes \pi_{X_{1}}^{*}\left(F_{*}\left(\theta^{-1}\right)\right)\right]=0\right\} / \sim .
\end{gathered}
$$

Proof. Consider a sheaf $\mathscr{E}$ over $X_{1} \times S$ with $\langle\mathscr{E}\rangle \in \underline{\mathcal{N}}_{X_{1}}^{s}(S)$. Then $\langle\mathscr{E}\rangle$ is an element of $\left[\mathscr{D} \times \mathcal{N}_{X_{1}}^{s} \underline{\mathcal{N}}_{X_{1}}^{s}\right](S)$ if and only if the classifying map $\Phi_{\mathcal{E}}: S \rightarrow \mathcal{N}_{X_{1}}^{s}$ factorizes as $S \stackrel{\varphi}{\longrightarrow} \mathscr{D} \subset \mathcal{N}_{X_{1}}^{s}$. By Lemma 5.4 (b) there exists a universal family $\varepsilon_{0}$ over $X_{1} \times \mathscr{D}$ and we have $\mathcal{E} \sim\left(\operatorname{id}_{X_{1}} \times \varphi\right)^{*} \mathcal{E}_{0}$. Since $\mathscr{D}$ is defined (over an étale cover) by a Fitting ideal and since the formation of the Fitting ideal commutes with any base change, we deduce that $\left[\mathscr{D} \times{ }_{\mathcal{N}_{X_{1}}^{s}}^{s} \underline{\mathcal{N}}_{X_{1}}^{s}\right](S)=\underline{D}(S)$. Since $\mathcal{N}_{X_{1}}^{s}$ universally corepresents the functor $\underline{\mathcal{N}}_{X_{1}}^{s}$, this shows that $\mathscr{D}$ corepresents the functor $\underline{D}$. The existence of a universal family $\varepsilon_{0}$ over $X \times \mathscr{D}$ implies that $\mathscr{D}$ represents the functor $\underline{D}$.

Proposition 5.6. There is a scheme-theoretical equality

$$
\tilde{D}=\psi^{-1} \mathscr{D} \text {. }
$$

Proof. In order to show that the subschemes $\tilde{D}$ and $\psi^{-1} \mathscr{D}$ of $J X_{1} \times Z$ coincide, it is enough to show that the two subsets $\operatorname{Mor}(S, \tilde{D})$ and $\operatorname{Mor}\left(S, \psi^{-1} \mathscr{D}\right)$ of $\operatorname{Mor}\left(S, J X_{1} \times\right.$ $Z)$ coincide for any $k$-scheme $S$. Consider $\Phi \in \operatorname{Mor}\left(S, J X_{1} \times Z\right)$ and denote $\mathcal{E}_{\Phi}:=\left(\operatorname{id}_{X_{1}} \times \Phi\right)^{*}(\mathcal{L} \otimes \mathcal{V})$. By definition of $\tilde{D}$ we have $\Phi \in \operatorname{Mor}(S, \tilde{D})$ if and only if $\operatorname{Fitt}_{4}\left[R^{1} \pi_{S *}\left(\mathcal{E}_{\Phi}^{*} \otimes \pi_{X_{1}}^{*}\left(F_{*}\left(\theta^{-1}\right)\right)\right)\right]=0$. On the other hand $\Phi \in \operatorname{Mor}\left(S, \psi^{-1}(\mathscr{D})\right)$ if and only if $\psi \circ \Phi \in \operatorname{Mor}(S, \mathcal{D})$. The latter set equals $\underline{D}(S)$ by Proposition 5.5. Since $(\psi \circ \Phi)^{*} \mathcal{E}_{0} \sim \mathcal{E}_{\Phi}$, we are done.

Proposition 5.7. There is a scheme-theoretical equality

$$
\mathcal{D}=\mathcal{Q} \text {. }
$$

Proof. We note that $\underline{D}(S)$ and $\underline{\mathcal{Q}}(S)$ are subsets of $\underline{\mathcal{N}}_{X_{1}}^{s}(S)$ (the injectivity of the $\operatorname{map} \underline{\mathcal{Q}}(S) \rightarrow \underline{\mathcal{N}}_{X_{1}}^{S}(S)$ is proved similarly as in the proof of Proposition 4.7). Since $\mathscr{D}$ and $\mathcal{Q}$ corepresent the two functors $\underline{\mathcal{D}}$ and $\underline{\mathcal{Q}}$, it will be enough to show that the set $\underline{D}(S)$ coincides with $\underline{Q}(S)$ for any $k$-scheme $S$.

We first show that $\underline{D}(S) \subset \underline{\mathcal{Q}}(S)$. Consider a sheaf $\mathcal{E}$ with $\langle\mathscr{E}\rangle \in \underline{D}(S)$. For simplicity we denote the sheaf $\mathcal{E}^{*} \otimes \pi_{X_{1}}^{*}\left(F_{*}\left(\theta^{-1}\right)\right)$ by $\mathcal{H}$. By [Ha], Theorem 12.11, there is an isomorphism

$$
R^{1} \pi_{S *} \mathscr{H} \otimes k(s) \cong H^{1}\left(X_{1} \times\{s\},\left.\mathcal{H}\right|_{X_{1} \times\{s\}}\right) \quad \text { for all } s \in S .
$$

From our assumption Fitt $4\left[R^{1} \pi_{S *} \mathcal{H}\right]=0$, we obtain $\operatorname{dim} H^{1}\left(X_{1} \times\{s\},\left.\mathcal{H}\right|_{X_{1} \times\{s\}}\right) \geq$ 5, or equivalently $\operatorname{dim} H^{0}\left(X_{1} \times\{s\},\left.\mathcal{H}\right|_{X_{1} \times\{s\}}\right) \geq 1$, i.e., the vector bundle $\left.\mathcal{E}\right|_{X_{1} \times\{s\}}$ 
is a subsheaf, hence by Proposition 3.1 (a) (ii) a subbundle, of $F_{*}\left(\theta^{-1}\right)$. This implies that the set-theoretical image of the classifying map $\Phi_{\mathcal{E}}$ is contained in $\operatorname{supp} \mathcal{Q}$. Taking connected components of $S$, we can assume that the image of $\Phi_{\mathcal{E}}$ is a point. Therefore we can apply Lemma 5.4: there exists a locally free sheaf $\varepsilon_{0}$ over $X_{1} \times \Delta$ such that $\mathcal{E} \sim\left(\operatorname{id}_{X_{1}} \times \varphi\right)^{*} \mathcal{E}_{0}$. For simplicity we write $\mathscr{H}_{0}=\mathcal{E}_{0}^{*} \otimes \pi_{X_{1}}^{*}\left(F_{*}\left(\theta^{-1}\right)\right)$. In particular $\mathscr{H}=\left(\operatorname{id}_{X_{1}} \times \varphi\right)^{*} \mathcal{H}_{0}$. Since the projection $\pi_{\Delta}: X_{1} \times \Delta \rightarrow \Delta$ is of relative dimension 1 , taking the higher direct image $R^{1} \pi_{\Delta *}$ commutes with the (not necessarily flat) base change $\varphi: S \rightarrow \Delta$ ([Ha], Proposition 12.5), i.e., there is an isomorphism

$$
\varphi^{*} R^{1} \pi_{\Delta *} \mathscr{H}_{0} \cong R^{1} \pi_{S *} \mathscr{H} .
$$

Since the formation of Fitting ideals also commutes with any base change (see [E], Corollary 20.5), we obtain

$$
\operatorname{Fitt}_{4}\left[R^{1} \pi_{S *} \mathscr{H}\right]=\operatorname{Fitt}_{4}\left[R^{1} \pi_{\Delta *} \mathcal{H}_{0}\right] \cdot \mathcal{O}_{S} .
$$

Since $\operatorname{Fitt}_{4}\left[R^{1} \pi_{S *} \mathscr{H}\right]$ is equal to 0 and $\mathcal{O}_{\Delta} \rightarrow \varphi_{*} \mathcal{O}_{S}$ is injective, we deduce that Fitt $_{4}\left[R^{1} \pi_{\Delta *} \mathscr{H}_{0}\right]=0$. Since by Proposition 3.1 (b) (iii) $\operatorname{dim} R^{1} \pi_{\Delta *} \mathscr{H}_{0} \otimes k\left(s_{0}\right)=5$ for the closed point $s_{0} \in \Delta$, we have Fitt ${ }_{5}\left[R^{1} \pi_{\Delta *} \mathcal{H}_{0}\right]=\mathcal{O}_{\Delta}$. We deduce by [E], Proposition 20.8, that the sheaf $R^{1} \pi_{\Delta *} \mathscr{H}_{0}$ is a free $A$-module of rank 5. By [Ha], Theorem 12.11 (b), we deduce that there is an isomorphism

$$
\pi_{\Delta *} \mathcal{H}_{0} \otimes k\left(s_{0}\right) \cong H^{0}\left(X_{1} \times s_{0},\left.\mathcal{H}\right|_{X_{1} \times\left\{s_{0}\right\}}\right)
$$

Again by Proposition 3.1 (b) (iii) we obtain $\operatorname{dim} \pi_{\Delta *} \mathscr{H}_{0} \otimes k\left(s_{0}\right)=1$. In particular the $\mathcal{O}_{\Delta}$-module $\pi_{\Delta *} \mathscr{H}_{0}$ is not zero and therefore there exists a nonzero global section $i \in H^{0}\left(\Delta, \pi_{\Delta *} \mathscr{H}_{0}\right)=H^{0}\left(X_{1} \times \Delta, \mathcal{E}_{0}^{*} \otimes \pi_{X_{1}}^{*} F_{*}\left(\theta^{-1}\right)\right)$. We pull-back $i$ under the

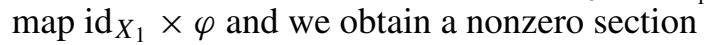

$$
j=\left(\operatorname{id}_{X_{1}} \times \varphi\right)^{*} i \in H^{0}\left(X_{1} \times S, \mathcal{E}^{*} \otimes \pi_{X_{1}}^{*} F_{*}\left(\theta^{-1}\right)\right) .
$$

Now we apply Lemma 4.3 and we continue as in the proof of Proposition 4.7. This shows that $\langle\mathcal{E}\rangle \in \underline{\mathcal{Q}}(S)$.

We now show that $\mathcal{Q}(S) \subset \mathscr{D}(S)$. Consider a sheaf $\mathscr{E} \in \mathbb{Q}(S)$. The nonzero global section $j \in H^{0}\left(X_{1} \times S, \overline{\mathscr{H}}\right)=H^{0}\left(S, \pi_{S *} \mathscr{H}\right)$ determines by evaluation at a point $s \in S$ an element $\alpha \in \pi_{S *} \mathscr{H} \otimes k(s)$. The image of $\alpha$ under the natural map

$$
\varphi^{0}(s): \pi_{S *} \mathscr{H} \otimes k(s) \longrightarrow H^{0}\left(X_{1} \times\{s\},\left.\mathcal{H}\right|_{X_{1} \times\{s\}}\right)
$$

coincides with $\left.j\right|_{X_{1 \times\{s\}}}$ which is nonzero. Also, as $\operatorname{dim} H^{0}\left(X_{1} \times\{s\},\left.\mathscr{H}\right|_{X_{1} \times\{s\}}\right)=1$, we obtain that $\varphi^{0}(s)$ is surjective. Hence by [Ha], Theorem 12.11 , the sheaf $R^{1} \pi_{S *} \mathcal{H}$ is locally free of rank 5. Again by [E], Proposition 20.8, this is equivalent to Fitt $_{4}\left[R^{1} \pi_{S *} \mathscr{H}\right]=0$ and Fitt $_{5}\left[R^{1} \pi_{S *} \mathscr{H}\right]=\mathcal{O}_{S}$ and we are done. 


\section{Chern class computations}

In this section we will compute the length of the determinantal subscheme $\mathscr{D} \subset \mathcal{N}_{X_{1}}$ by evaluating the Chern class $c_{5}\left(\mathcal{F}_{0}-\mathscr{F}_{1}\right)-$ see Proposition $5.1(\mathrm{~b})$.

Let $l$ be a prime number different from $p$. We have to recall some properties of the cohomology ring $H^{*}\left(X_{1} \times J X_{1} \times Z, \mathbb{Z}_{l}\right)$ (see also [LN]). In the sequel we identify all classes of $H^{*}\left(X_{1}, \mathbb{Z}_{l}\right), H^{*}\left(J X_{1}, \mathbb{Z}_{l}\right)$ etc. with their preimages in $H^{*}\left(X_{1} \times J X_{1} \times Z, \mathbb{Z}_{l}\right)$ under the natural pull-back maps.

Let $\Theta \in H^{2}\left(J X_{1}, \mathbb{Z}_{l}\right)$ denote the class of the theta divisor in $J X_{1}$. Let $f$ denote a positive generator of $H^{2}\left(X_{1}, \mathbb{Z}_{l}\right)$. The cup product $H^{1}\left(X_{1}, \mathbb{Z}_{l}\right) \times H^{1}\left(X_{1}, \mathbb{Z}_{l}\right) \rightarrow$ $H^{2}\left(X_{1}, \mathbb{Z}_{l}\right) \simeq \mathbb{Z}_{l}$ gives a symplectic structure on $H^{1}\left(X_{1}, \mathbb{Z}_{l}\right)$. Choose a symplectic basis $e_{1}, e_{2}, e_{3}, e_{4}$ of $H^{1}\left(X_{1}, \mathbb{Z}_{l}\right)$ such that $e_{1} e_{3}=e_{2} e_{4}=-f$ and all other products $e_{i} e_{j}=0$. We can then normalize the Poincaré bundle $\mathcal{L}$ on $X_{1} \times J X_{1}$ so that

$$
c(\mathcal{L})=1+\xi_{1}
$$

where $\xi_{1} \in H^{1}\left(X_{1}, \mathbb{Z}_{l}\right) \otimes H^{1}\left(J X_{1}, \mathbb{Z}_{l}\right) \subset H^{2}\left(X_{1} \times J X_{1}, \mathbb{Z}_{l}\right)$ can be written as

$$
\xi_{1}=\sum_{i=1}^{4} e_{i} \otimes \varphi_{i}
$$

with $\varphi_{i} \in H^{1}\left(J X_{1}, \mathbb{Z}_{l}\right)$. Moreover, we have by the same reasoning, applying [ACGH], p. 335 and p. 21,

$$
\xi_{1}^{2}=-2 \Theta f \quad \text { and } \quad \Theta^{2}\left[J X_{1}\right]=2 .
$$

Since the variety $\mathcal{M}_{X_{1}}(x)$ is a smooth intersection of 2 quadrics in $\mathbb{P}^{5}$, one can work out that the $l$-adic cohomology groups $H^{i}\left(\mathcal{M}_{X_{1}}(x), \mathbb{Z}_{l}\right)$ for $i=0, \ldots, 6$ are (see e.g. [Re], p. 0.19)

$$
\mathbb{Z}_{l}, \quad 0, \quad \mathbb{Z}_{l}, \quad \mathbb{Z}_{l}^{4}, \quad \mathbb{Z}_{l}, \quad 0, \quad \mathbb{Z}_{l} .
$$

In particular $H^{2}\left(\mathcal{M}_{X_{1}}(x), \mathbb{Z}_{l}\right)$ is free of rank 1 and, if $\alpha$ denotes a positive generator of it, then

$$
\alpha^{3}\left[\mathcal{M}_{X_{1}}(x)\right]=4 .
$$

According to [N2] p. 338 and applying reduction mod p and a comparison theorem, the Chern classes of the universal bundle $\mathcal{U}$ are of the form

$$
c_{1}(\mathcal{U})=\alpha+f \quad \text { and } \quad c_{2}(U)=\chi+\xi_{2}+\alpha f
$$

with $\chi \in H^{4}\left(\mathcal{M}_{X_{1}}(x), \mathbb{Z}_{l}\right)$ and $\xi_{2} \in H^{1}\left(X_{1}, \mathbb{Z}_{l}\right) \otimes H^{3}\left(\mathcal{M}_{X_{1}}(x), \mathbb{Z}_{l}\right)$. As in [N2] and $[\mathrm{KN}]$ we write

$$
\beta=\alpha^{2}-4 \chi \quad \text { and } \quad \xi_{2}^{2}=\gamma f \quad \text { with } \gamma \in H^{6}\left(\mathcal{M}_{X_{1}}(x), \mathbb{Z}_{l}\right) .
$$


Then the relations of $[\mathrm{KN}]$ give

$$
\alpha^{2}+\beta=0 \quad \text { and } \quad \alpha^{3}+5 \alpha \beta+4 \gamma=0 .
$$

Hence $\beta=-\alpha^{2}, \gamma=\alpha^{3}$. Together with (12) and (13) this gives

$$
c_{2}(U)=\frac{\alpha^{2}}{2}+\xi_{2}+\alpha f \quad \text { and } \quad \xi_{2}^{2}=\alpha^{3} f
$$

Define $\Lambda \in H^{1}\left(J X_{1}, \mathbb{Z}_{l}\right) \otimes H^{3}\left(\mathcal{M}_{X_{1}}(x), \mathbb{Z}_{l}\right)$ by

$$
\xi_{1} \xi_{2}=\Lambda f
$$

Then we have for dimensional reasons and noting that $H^{5}\left(\mathcal{M}_{X_{1}}(x), \mathbb{Z}_{l}\right)=0$, that the following classes are all zero:

$$
f^{2}, \quad \xi_{1}^{3}, \quad \alpha^{4}, \quad \xi_{1} f, \quad \xi_{2} f, \quad \alpha \xi_{2}, \quad \alpha \Lambda, \quad \Theta^{2} \Lambda, \quad \Theta^{3} .
$$

Finally, $Z$ is the $\mathbb{P}^{1}$-bundle associated to the vector bundle $U_{x}$ on $\mathcal{M}_{X_{1}}(x)$. Let $H \in H^{2}\left(Z, \mathbb{Z}_{l}\right)$ denote the first Chern class of the tautological line bundle on $Z$. We have, using the definition of the Chern classes $c_{i}(U)$ and (11),

$$
H^{2}=\alpha H-\frac{\alpha^{2}}{2}, \quad H^{4}=0, \quad \alpha^{3} H[Z]=4
$$

and we get for the "universal" bundle $\mathcal{V}$,

$$
c_{1}(\mathcal{V})=\alpha \quad \text { and } \quad c_{2}(\mathcal{V})=\frac{\alpha^{2}}{2}+\xi_{2}+H f
$$

Lemma 6.1. (a) The cohomology class $\alpha \cdot c_{5}\left(\mathcal{F}_{0}-\mathcal{F}_{1}\right) \in H^{12}\left(J X_{1} \times Z, \mathbb{Z}_{l}\right)$ is a multiple of the class $\alpha^{3} H \Theta^{2}$.

(b) The pull-back under the map $\varphi: Z \longrightarrow \mathcal{M}_{X_{1}} \cong \mathbb{P}^{3}$ of the class of a point is the class $H^{3}=\frac{\alpha^{2}}{2} H-\frac{\alpha^{3}}{2}$.

Proof. For part (a) it is enough to note that all other relevant cohomology classes vanish, since $\alpha^{4}=0$ and $\alpha \Lambda=0$.

As for part (b), it suffices to show that $c_{1}\left(\varphi^{*} \mathcal{O}_{\mathbb{P}^{3}}(1)\right)=H$. The line bundle $\mathcal{O}_{\mathbb{P}^{3}}(1)$ is the inverse of the determinant line bundle $[\mathrm{KM}]$ over the moduli space $\mathcal{M}_{X_{1}}$. Since the formation of the determinant line bundle commutes with any base change (see $[\mathrm{KM}]$ ), the pull-back $\varphi^{*} \mathcal{O}_{\mathbb{P}^{3}}(1)$ is the inverse of the determinant line bundle associated to the family $\mathcal{V} \otimes \pi_{X_{1}}^{*} N$ for any line bundle $N$ of degree 1 over $X_{1}$. Hence the first Chern class of $\varphi^{*} \mathcal{O}_{\mathbb{P}^{3}}(1)$ can be computed by the Grothendieck-Riemann-Roch theorem applied to the sheaf $\mathcal{V} \otimes \pi_{X_{1}}^{*} N$ over $X_{1} \times Z$ and the morphism 
$\pi_{Z}: X_{1} \times Z \rightarrow Z$. We have

$$
\begin{aligned}
\operatorname{ch}\left(\mathcal{V} \otimes \pi_{X_{1}}^{*} N\right) \cdot \pi_{X_{1}}^{*} t d\left(X_{1}\right) & =\left(2+\alpha+\left(-\xi_{2}-H f\right)+\text { h.o.t. }\right)(1+f)(1-f) \\
& =2+\alpha+\left(-\xi_{2}-H f\right)+\text { h.o.t., }
\end{aligned}
$$

and therefore G-R-R implies that $c_{1}\left(\varphi^{*} \mathcal{O}_{\mathbb{P}^{3}}(1)\right)=H$ - note that $\pi_{Z *}\left(\xi_{2}\right)=0$.

Proposition 6.2. We have

$$
l(\mathscr{D})=\frac{1}{24} p^{3}\left(p^{2}-1\right)
$$

Proof. Let $\lambda$ denote the length of the subscheme $m^{-1}(\mathscr{D}) \subset J X_{1} \times \mathcal{M}_{X_{1}}$ Since the map $m^{s}$ is étale of degree 16 , we obviously have the relation $\lambda=16 \cdot l(\mathscr{D})$. According to Lemma 6.1 (b) we have in $H^{10}\left(J X_{1} \times Z, \mathbb{Z}_{l}\right)$

$$
\left[(\mathrm{id} \times \varphi)^{-1}(p t)\right]=H^{3} \cdot \frac{\Theta^{2}}{2}=\frac{1}{4} \alpha^{2} H \Theta^{2}-\frac{1}{4} \alpha^{3} \Theta^{2},
$$

where $p t$ denotes the class of a point in $J X_{1} \times \mathcal{M}_{X_{1}}$. Using Proposition 5.6 we obtain that the class $\delta=c_{5}\left(\mathscr{F}_{0}-\mathcal{F}_{1}\right) \in H^{10}\left(J X_{1} \times Z, \mathbb{Z}_{l}\right)$ equals $\lambda \cdot\left(\frac{1}{4} \alpha^{2} H \Theta^{2}-\frac{1}{4} \alpha^{3} \Theta^{2}\right)$. Intersecting with $\alpha$ we obtain with Lemma 6.1 (a) and (16)

$$
\alpha \cdot c_{5}\left(\mathscr{F}_{0}-\mathscr{F}_{1}\right)=\frac{\lambda}{4} \alpha^{3} H \Theta^{2} .
$$

So we have to compute the class $\alpha \cdot c_{5}\left(\mathcal{F}_{0}-\mathcal{F}_{1}\right)$. By (9) and (10),

$$
\operatorname{ch}(\mathcal{L})=1+\xi_{1}-\Theta f
$$

whereas by (14), (16) and (18),

$$
\operatorname{ch}(\mathcal{V})=2+\alpha+\left(-\xi_{2}-H f\right)+\frac{1}{12}\left(-\alpha^{3}-6 \alpha H f\right)+\frac{1}{12}\left(\alpha^{3} f-\alpha^{2} H f\right) .
$$

Moreover

$$
\operatorname{ch}\left(\pi_{X_{1}}^{*}\left(F_{*}\left(\theta^{-1}\right) \otimes \omega_{X_{1}}\right)\right) \cdot \pi_{X_{1}}^{*} t d\left(X_{1}\right)=p+(2 p-2) f .
$$

So using (14), (15) and (16),

$$
\begin{aligned}
c h\left(\mathcal{V}^{*} \otimes\right. & \left.\mathcal{L}^{*} \otimes \pi_{X_{1}}^{*}\left(F_{*}\left(\theta^{-1}\right) \otimes \omega_{X_{1}}\right)\right) \cdot \pi_{X_{1}}^{*} t d\left(X_{1}\right) \\
=2 p & +\left[(4 p-4) f-p \alpha-2 p \xi_{1}\right] \\
& +\left[p \alpha \xi_{1}-2 p \Theta f-(2 p-2) \alpha f-p \xi_{2}-p H f\right] \\
& +\left[\frac{p}{12} \alpha^{3}+\frac{p}{2} \alpha H f+p \Lambda f+p \alpha \Theta f\right] \\
& +\left[\frac{3 p-2}{12} \alpha^{3} f-\frac{p}{12} \alpha^{3} \xi_{1}-\frac{p}{12} \alpha^{2} H f\right]+\left[-\frac{p}{12} \alpha^{3} \Theta f\right] .
\end{aligned}
$$


Hence by Grothendieck-Riemann-Roch for the morphism $q$ we get

$$
\begin{aligned}
\operatorname{ch}\left(\mathcal{F}_{1}\right)=4 p-4 & +[-(2 p-2) \alpha-2 p \Theta-p H]+\left[\frac{p}{2} \alpha H+p \Lambda+p \alpha \Theta\right] \\
+ & {\left[\frac{3 p-2}{12} \alpha^{3}-\frac{p}{12} \alpha^{2} H\right]+\left[-\frac{p}{12} \alpha^{3} \Theta\right] . }
\end{aligned}
$$

From (10) and (18) we easily obtain

$$
\operatorname{ch}\left(\mathcal{F}_{0}\right)=4 p-2 p \alpha+\frac{p}{6} \alpha^{3} .
$$

So

$$
\begin{aligned}
\operatorname{ch}\left(\mathcal{F}_{0}-\mathcal{F}_{1}\right)=4 & +[2 p \Theta-2 \alpha+p H]+\left[-\frac{p}{2} \alpha H-p \Lambda-p \alpha \Theta\right] \\
+ & {\left[-\frac{p+1}{12} \alpha^{3}+\frac{p}{12} \alpha^{2} H\right]+\left[\frac{p}{12} \alpha^{3} \Theta\right] . }
\end{aligned}
$$

Defining $p_{n}:=n ! \cdot c h_{n}\left(\mathcal{F}_{0}-\mathcal{F}_{1}\right)$ we have according to Newton's recursive formula ([F] p. 56),

$c_{5}\left(\mathcal{F}_{0}-\mathcal{F}_{1}\right)=\frac{1}{5}\left(p_{5}-\frac{5}{6} p_{2} p_{3}-\frac{5}{4} p_{1} p_{4}+\frac{5}{6} p_{1}^{2} p_{3}+\frac{5}{8} p_{1} p_{2}^{2}-\frac{5}{12} p_{1}^{3} p_{2}+\frac{1}{24} p_{1}^{5}\right)$

with

$$
\begin{gathered}
p_{1}=2 p \Theta-2 \alpha+p H, \quad p_{2}=-p(\alpha H+2 \Lambda+2 \alpha \Theta), \\
p_{3}=\frac{1}{2}\left(-(p+1) \alpha^{3}+p \alpha^{2} H\right), \quad p_{4}=2 p \alpha^{3} \Theta, \quad p_{5}=0 .
\end{gathered}
$$

Now an immediate computation using (16) and (17) gives

$$
\alpha \cdot c_{5}\left(\mathcal{F}_{0}-\mathcal{F}_{1}\right)=\frac{p^{3}\left(p^{2}-1\right)}{6} \alpha^{3} H \Theta^{2} .
$$

We conclude from (19) that $\lambda=\frac{2}{3} p^{3}\left(p^{2}-1\right)$ and we are done.

Remark 6.3. If $k=\mathbb{C}$, the number of maximal subbundles of a general vector bundle has recently been computed by Y. Holla by using Gromov-Witten invariants [Ho]. His formula ([Ho], Corollary 4.6) coincides with ours.

\section{Proof of Theorem 2}

The proof of Theorem 2 is now straightforward. It suffices to combine Corollary 4.9, Proposition 5.7 and Proposition 6.2 to obtain the length $l(\mathscr{B})$.

The fact that $\mathscr{B}$ is a local complete intersection follows from the isomorphism $\mathscr{B}_{\theta}=\mathcal{Q}_{0}$ (Proposition 4.6) and Proposition 4.1. 


\section{Questions and remarks}

(1) Is the rank- $p$ vector bundle $F_{*} L$ very stable, i.e. $F_{*} L$ has no nilpotent $\omega_{X_{1}}$-valued endomorphisms, for a general line bundle?

(2) Is $F_{*}\left(\theta^{-1}\right)$ very stable for a general curve $X$ ? Note that very-stability of $F_{*}\left(\theta^{-1}\right)$ implies reducedness of $\mathscr{B}$ (see e.g. [LN], Lemma 3.3).

(3) If $g=2$, we have shown that for a general stable $E \in \mathcal{M}_{X}$ the fibre $V^{-1}(E)$ consists of $\frac{1}{3} p\left(p^{2}+2\right)$ stable vector bundles $E_{1} \in \mathcal{M}_{X_{1}}$, i.e. bundles $E_{1}$ such that $F^{*} E_{1} \cong E$ or equivalently (via adjunction) $E_{1} \subset F_{*} E$. The Quot-scheme parametrizing rank-2 subbundles of degree 0 of the rank- $2 p$ vector bundle $F_{*} E$ has expected dimension 0 , contains the fibre $V^{-1}(E)$, but it also has a 1-dimensional component arising from Frobenius-destabilized bundles as follows: for any $M \in \operatorname{Pic}^{1}(X)$ with $\operatorname{Hom}\left(M^{-1}, E\right) \neq 0$ consider a stable degree 0 rank-2 bundle $E_{1}$ such that $F^{*} E_{1}$ has a nonzero map to $M^{-1}$.

(4) If $p=3$ the base locus $\mathcal{B}$ consists of 16 reduced points, which correspond to the 16 nodes of the Kummer surface associated to $J X$ (see [LP2], Corollary 6.6). For general $p$, does the configuration of points determined by $\mathscr{B}$ have some geometric significance?

\section{Appendix on base loci and substack of non-semistable vector bundles.}

For lack of a suitable reference, we include a detailed proof of the following fact, which was used in Lemma 4.5. We use the notation of Lemma 4.5.

Proposition A. Let X be a smooth curve of genus 2 . The closed substack $\mathfrak{M}_{X}^{1}$ equals the base locus $\mathrm{Bs}|\mathcal{O}(1)|$ of the linear system $|\mathcal{O}(1)|$ over the moduli stack $\mathfrak{M}_{X}^{\leq 1}$.

Proof. Let $E$ be a rank-2 vector bundle with trivial determinant over $X$. It follows from [R], Proposition 1.6.2, that $E$ is semistable if and only if there exists a line bundle $M$ of degree 1 such that $h^{0}(X, E \otimes M)=h^{1}(X, E \otimes M)=0$. Consider the determinant divisor $\theta_{M}$ associated to $M$. Then $\theta_{M} \in|\mathcal{O}(1)|$ and for an $S$-valued point $\varepsilon$ of $\mathfrak{M}_{X}^{\leq 1}$

$$
\operatorname{supp}\left(\theta_{M}\right)=\left\{s \in S \mid h^{0}\left(X, \mathcal{E}_{s} \otimes M\right)>0\right\} .
$$

We know (see e.g. [B1], Proposition 2.5) that the linear system $|\mathcal{O}(1)|$ is linearly generated by the divisors $\theta_{M}$ when $M$ varies in $\operatorname{Pic}^{1}(X)$. The previous equivalence implies that the open complements of the closed substacks $\mathrm{Bs}|\mathcal{O}(1)|$ and $\mathfrak{M}_{X}^{1}$ coincide. To conclude the proposition it remains to show that the base locus $\mathrm{Bs}|\mathcal{O}(1)|$ is a reduced substack of $\mathfrak{M}_{X}^{\leq 1}$. 
The normal bundle $N$ of the closed substack $\mathfrak{M}_{X}^{1}$ in $\mathfrak{M}_{X}^{\leq 1}$ can be described as follows(e.g. [He], Behauptung 2.1.12, p. 44 or [VL], exposé 4, Théorème 4, p. 90): let $\&$ denote the universal bundle over $X \times \mathfrak{M}_{X}$ restricted to $X \times \mathfrak{M}_{X}^{1}$. There is a canonical inclusion

$$
\operatorname{End}_{0}(\&)^{\text {filt }} \subset \operatorname{End}_{0}(\mathscr{E}),
$$

where $\operatorname{End}_{0}(\varepsilon)^{\text {filt }}$ denotes the sheaf of tracefree endomorphisms preserving the Harder-Narasimhan filtration. We denote by $\operatorname{End}_{0}^{\prime}(\varepsilon)$ the quotient. Then the normal bundle $N$ equals $R^{1} p_{*} \operatorname{End}_{0}^{\prime}(\mathcal{E})$, where $p$ denotes projection onto $\mathfrak{M}_{X}^{1}$. In the rank-2 case the universal Harder-Narasimhan filtration over $X \times \mathfrak{M}_{X}^{1}$ is of the form

$$
0 \longrightarrow \mathcal{L} \longrightarrow \mathcal{E} \longrightarrow \mathcal{L}^{-1} \longrightarrow 0,
$$

where $\mathcal{L}$ is a degree 1 line bundle. In that case we have $\operatorname{End}_{0}^{\prime}(\mathscr{E})=\operatorname{Hom}\left(\mathcal{L}, \mathcal{L}^{-1}\right)$ and therefore $N=R^{1} p_{*} \mathcal{L}^{-2}$.

Consider an $S$-point $\mathscr{E} \in \mathfrak{M}_{X}^{\leq 1}(S)$ and $x \in S$ such that the vector bundle $\mathcal{E}_{x}=$ $E \in \mathfrak{M}_{X}^{1}(k)$, i.e., $E$ is destabilized by $L$ of degree 1 . Consider a line bundle $M$ of degree 1 and its associated determinant divisor $\theta_{M}$. Then the divisor $\theta_{M}$ contains the closed substack $\mathfrak{M}_{X}^{1}$. The Kodaira-Spencer map at the point $x \in S$ associated to $\mathscr{E}$ is a $k$-linear map

$$
\kappa: T_{x} S \longrightarrow H^{1}\left(X, \operatorname{End}_{0}(E)\right) .
$$

Note that we consider bundles with trivial determinant, hence $\kappa$ takes values in $H^{1}\left(X, \operatorname{End}_{0}(E)\right)$. By [Las], Sections II and III, the linear form on $T_{x} S$ defining the tangent space $T_{x} \theta_{M}$ to the determinant divisor $\theta_{M}$ is the map $\Phi \circ \kappa$, where $\Phi$ is given by cup product

$$
\Phi: H^{1}\left(X, \operatorname{End}_{0}(E)\right) \longrightarrow \operatorname{Hom}\left(H^{0}(X, E \otimes M), H^{1}(X, E \otimes M)\right), \quad e \mapsto \cup e .
$$

Using Serre duality we identify $H^{1}\left(X, \operatorname{End}_{0}(E)\right)^{*}$ with $H^{0}\left(X, \operatorname{End}_{0}(E) \otimes \omega\right)$ and $H^{1}(X, E \otimes M)$ with $H^{0}\left(X, E \otimes \omega M^{-1}\right)^{*}$. The dual of $\Phi$ equals the symmetrized multiplication map of global sections (note that $\operatorname{End}_{0}(E)=\operatorname{Sym}^{2} E$ and $E=E^{*}$ )

$$
\mu: H^{0}(X, E \otimes M) \otimes H^{0}\left(X, E \otimes \omega M^{-1}\right) \longrightarrow H^{0}\left(X, \operatorname{End}_{0}(E) \otimes \omega\right) .
$$

Note that both spaces on the left have dimension equal to 1 for general $M$ and that $H^{0}(X, E \otimes M)=H^{0}(X, L \otimes M)$ and $H^{0}\left(X, E \otimes \omega M^{-1}\right)=H^{0}\left(X, L \otimes \omega M^{-1}\right)$ for general $M$. This implies that $\operatorname{dim} \operatorname{im}(\mu)=1$ and

$$
\operatorname{im}(\mu) \subset H^{0}\left(X, L^{2} \omega\right) \subset H^{0}\left(X, \operatorname{End}_{0}(E) \otimes \omega\right) .
$$

We denote by $h$ a generator of $\operatorname{im}(\mu)$. We obtain that for general $M$ the conormal vector defined by $T_{x} \theta_{M}$ is given (up to a scalar) by

$$
h \in H^{0}\left(X, L^{2} \omega\right)=H^{1}\left(X, L^{-2}\right)^{*}=N_{x}^{*} .
$$


The corresponding rational map

$$
\operatorname{Pic}^{1}(X) \longrightarrow \mathbb{P} H^{0}\left(X, L^{2} \omega\right)=\mathbb{P}^{2}, \quad M \mapsto h,
$$

is easily seen to be dominant. In particular its image is non degenerate. This shows that the point $E$ is a reduced point of $\mathrm{Bs}|\mathcal{O}(1)|$, because the linear span of the family of conormal vectors defined by $T_{x} \theta_{M}$ when $M$ varies in an open set of $\operatorname{Pic}^{1}(X)$ equals the full space $N_{x}^{*}$.

\section{References}

[ACGH] E. Arbarello, M. Cornalba, P. A. Griffiths, J. Harris, Geometry of Algebraic Curves. Grundlehren Math. Wiss. 267, Springer-Verlag, New York 1985. Zbl 0559.14017 MR 0770932

[B1] A. Beauville, Fibrés de rang 2 sur les courbes, fibré déterminant et fonctions thêta. Bull. Soc. Math. France 116 (1988), 431-448. Zbl 0691.14016 MR 1005388

[B2] A. Beauville, On the stability of the direct image of a generic vector bundle. Preprint. http://math.unice.fr/ beauvill/pubs/imdir.pdf

[BL] A. Beauville, Y. Laszlo, Conformal blocks and generalized theta functions. Comm. Math. Phys. 164 (1994), 385-419. Zbl 0815.14015 MR 1289330

[E] D. Eisenbud, Commutative Algebra. Grad. Texts in Math. 150, Springer-Verlag, Berlin 1994. Zbl 0819.13001 MR 1322960

[F] W. Fulton, Intersection Theory. Ergeb. Math. Grenzgeb. (3) 2, Springer-Verlag, Berlin 1984. Zbl 0541.14005 MR R0732620

[G] A. Grothendieck, Fondements de la Géométrie Algébrique, IV, Les schémas de Hilbert. Séminaire Bourbaki, t. 13, 1960/61, n. 221. Zbl 0239.14002 MR 146040

[Ha] R. Hartshorne, Algebraic Geometry. Grad. Texts in Math. 52, Springer-Verlag, New York, Heidelberg, Berlin 1977. Zbl 0367.14001 MR 0463157

[Hir] A. Hirschowitz, Problèmes de Brill-Noether en rang supérieur. C. R. Acad. Sci. 307 (1988), 153-156. Zbl 0654.14017 MR 0956606

[He] J. Heinloth, Über den Modulstack der Vektorbündel auf Kurven, Diploma Thesis. http://www.uni-due.de/ hm0002/

[Ho] Y. I. Holla, Counting maximal subbundles via Gromov-Witten invariants. Math. Ann. 328 (2004), 121-133. Zbl 1065.14042 MR 2030371

[HL] D. Huybrechts, M. Lehn, The geometry of moduli spaces of sheaves. Aspects Math E31, Vieweg \& Sohn, Braunschweig 1997. Zbl 0872.14002 MR 1450870

[JRXY] K. Joshi, S. Ramanan, E.Z. Xia, J.-K. Yu, On vector bundles destabilized by Frobenius pull-back. Compositio Math. 142 (2006), 616-630. Zbl 1101.14049 MR 2231194

[K] N. Katz, Nilpotent connections and the monodromy theorem: Applications of a result of Turittin. Inst. Hautes Études Sci. Publ. Math. 39 (1970), 175-232. Zbl 0221.14007 MR 0291177 
[KN] A. King, P. E. Newstead, On the cohomology ring of the moduli space of rank 2 vector bundles on a curve. Topology 37 (1998), 407-418. Zbl 0913.14008 MR 1489212

[KM] F. Knudsen, D. Mumford, The projectivity of the moduli space of stable curves I. Math. Scand. 39 (1976), 19-55. Zbl 0343.14008 MR 0437541

[L] H. Lange, Some geometrical aspects of vector bundles on curves. In Topics in algebraic geometry, Aportaciones Mat. Notas Investigación 5, Soc. Mat. Mexicana, México 1992, 53-74. Zbl 0899.14012 MR 1308330

[LS] H. Lange, U. Stuhler, Vektorbündel auf Kurven und Darstellungen der algebraischen Fundamentalgruppe. Math. Z. 156 (1977), 73-83. Zbl 0349.14018 MR 0472827

[LN] H. Lange, P. Newstead, Maximal subbundles and Gromov-Witten invariants. In A Tribute to C. S. Seshadri, Trends Math., Birkhäuser, Basel 2003, 310-322. Zbl 1071.14036 MR 2017590

[La1] A. Langer, Moduli spaces of sheaves in mixed characteristic. Duke Math. J. 124(2004), 571-586. Zbl 02113314 MR 2085175

[La2] A. Langer, Moduli spaces and Castelnuovo-Mumford regularity of sheaves on surfaces. Amer. J. Math. 128 (2006), 373-417. Zbl 1102.14030 MR 2214897

[Las] Y. Laszlo, Un théorème de Riemann pour les diviseurs thêta sur les espaces de modules de fibrés stables sur une courbe. Duke Math. J. 64 (1991), 333-347. Zbl 0753.14023 MR 1136379

[LP1] Y. Laszlo, C. Pauly, The action of the Frobenius map on rank 2 vector bundles in characterictic 2. J. Algebraic Geom. 11 (2002), 219-243. Zbl 1080.14527 MR 1874113

[LP2] Y. Laszlo, C. Pauly, The Frobenius map, rank 2 vector bundles and Kummer's quartic surface in characteristic 2 and 3. Adv. Math. 185 (2004), 246-269. Zbl 1055.14038 MR 2060469

[MS] V. B. Mehta, S. Subramanian, Nef line bundles which are not ample. Math. Z. 219 (1995), 235-244. Zbl 0826.14009 MR 1337219

[Mo] S. Mochizuki, Foundations of p-adic Teichmüller Theory. AMS/IP Stud. Adv. Math. 11, Amer. Math. Soc., Providence, RI, 1999 Zbl 0969.14013 MR 1700772

[Mu S. Mukai, Non-Abelian Brill-Noether theory and Fano 3-folds. Sugaku 49 (1997), 1-24 (in Japanese; English transl. Sugaku Expositions 14 (2001), 125-153. Zbl 0929.14021 MR 1478148

[MuSa] S. Mukai, F. Sakai, Maximal subbundles of vector bundles on a curve. Manuscripta Math. 52 (1985), 251-256. Zbl 0572.14008 MR 0790801

[Mum] D. Mumford, The red book of varieties and schemes. Lecture Notes in Math. 1358, Springer-Verlag, Berlin 1999. Zbl 0658.14001 MR 1748380

[NR1] M. S. Narasimhan, S. Ramanan, Deformations of the moduli space of vector bundles over an algebraic curve. Ann. of Math. (2) 101 (1975), 391-417. Zbl 0314.14004 MR 0384797

[NR2] M. S. Narasimhan, S. Ramanan, Moduli of vector bundles on a compact Riemann surface. Ann. of Math. (2) 89 (1969), 14-51. Zbl 0186.54902 MR 0242185

[N1] P. E. Newstead, Stable bundles of rank 2 and odd degree over a curve of genus 2. Topology 7 (1968), 205-215. Zbl 0174.52901 MR 0237500 
[N2] P. E. Newstead, Characteristic classes of stable bundles of rank 2 over an algebraic curve. Trans. Amer. Math. Soc. 169 (1972), 337-345. Zbl 0256.14008 MR 0316452

[O1] B. Osserman, The generalized Verschiebung map for curves of genus 2. Math. Ann. 336 (2006), 963-986. Zbl 1111.14031 MR 2255181

[O2] B. Osserman, Mochizuki's crys-stable bundles: A lexicon and applications. Publ. Res. Inst. Math. Sci. 43 (2007), 95-119. MR 2317114

[R] M. Raynaud, Sections des fibrés vectoriels sur une courbe. Bull. Soc. Math. France 110 (1982), 103-125. Zbl 0505.14011 MR 0662131

[Re] M. Reid, The complete intersection of two or more quadrics, Doctoral Thesis, Cambridge University, Cambridge 1972.

[S] C. S. Seshadri, Vector bundles on curves. In Linear algebraic groups and their representations, Contemp. Math. 153, Amer. Math. Soc., Providence, RI, 1993, 163-200. Zbl 0799.14013 MR 1247504

[Sh] S. Shatz, The decomposition and specialization of algebraic families of vector bundles. Compositio Math. 35 (1977), 163-187. Zbl 0371.14010 MR 0498573

[SB] N. I. Shepherd-Barron, Semi-stability and reduction mod p. Topology 37 (1998), 659-664. Zbl 0926.14021 MR 1604907

[VL] J.-L. Verdier, J. Le Potier (eds.), Module des fibrés stables sur les courbes algébriques. Progr. Math. 54, Birkhäuser, Boston, MA, 1985 Zbl 0546.00011 MR 0790317

Received September 23, 2003; revised October 6, 2005

Herbert Lange, Mathematisches Institut, Universität Erlangen-Nürnberg,

Bismarckstrasse 1 1/2, 91054 Erlangen, Germany

E-mail: lange@mi.uni-erlangen.de

Christian Pauly, Département de Mathématiques, Université de Montpellier II,

Case Courrier 051, Place Eugène Bataillon, 34095 Montpellier Cedex 5, France

E-mail: pauly@math.univ-montp2.fr 